\title{
Do Eclipse Solar de 1919 ao Espetáculo das Lentes Gravitacionais
}

\author{
From Solar Eclipse of 1919 to the Spectacle of Gravitational Lensing \\ José Ademir Sales Lima*1], Rose Clivia Santos ${ }^{2}$ \\ ${ }^{1}$ Universidade de São Paulo, Departamento de Astronomia, São Paulo, SP, Brasil \\ ${ }^{2}$ Universidade Federal de São Paulo, Campus Diadema, Departamento de Física, Diadema, SP, Brasil
}

Recebido em 08 de Agosto de 2019. Aceito em 11 de Agosto de 2019.

\begin{abstract}
Um século depois de observada a deflexão da luz emitida pelas estrelas distantes, durante o eclipse solar de 1919, é interessante saber os conceitos que emergiram do experimento e suas consequências teóricas e observacionais para a cosmologia e astrofísica moderna. Além de confirmar a teoria gravitacional de Einstein, o maior legado foi a construção de uma nova área de pesquisa na ciência do cosmos, conhecida como lentes gravitacionais. A formação e magnificação de imagens múltiplas (miragens) pelo campo gravitacional de uma lente compacta ou extensa, estão entre os mais impressionantes fenômenos da natureza. Neste artigo apresentamos uma visão pedagógica do primeiro efeito genuíno de lente gravitacional, o quasar duplo QSO 0957+561. Descrevemos a formação de anéis, dos arcos gigantes, pequenos arcos e imagens múltiplas de Supernovas. É também surpreendente que a constante de Hubble e a quantidade de matéria escura no Universo possam ser mensuradas pela mesma técnica. Finalmente, o lenteamento de ondas gravitacionais, um efeito possível mas ainda não detectado, será também brevemente discutido.
\end{abstract}

Palavras-chave: Lentes Gravitacionais, Matéria Escura, Supernovas, Ondas Gravitacionais.

A century after observing the deflection of light emitted by distant stars during the solar eclipse of 1919, it is interesting to know the concepts emerged from the experiment and the theoretical and observational consequences for modern astrophysics and cosmology. In addition to confirming Einstein's gravitational theory, its greatest legacy was the construction of a new research area to cosmos science dubbed gravitational lensing. The formation and magnification of multiple images (mirages) by the gravitational field of a compact or extended lens are among the most striking phenomena of nature. This article presents a pedagogical view of the first genuine gravitational lens effect, the double quasar QSO $0957+561$. We also describe the formation of rings, giant arcs, arclets and multiple Supernova images. It is also surprising that the Hubble constant and the amount of dark matter in the Universe might be estimated by the same technique. Finally, the lensing of gravitational waves, a possible but still not yet detected effect, is also briefly discussed.

Keywords: Gravitational Lensing, Dark Matter, Supernovas, Gravitational Waves.

\section{Introdução}

O efeito de lente gravitacional (LG) refere-se a deflexão da luz pelo campo gravitacional da distribuição de matéria no Universo. Sua descrição pressupõe a existência do observador e de uma fonte luminosa distante, intercalados por uma lente gravitacional. A lente defletora pode ser um único astro ou um conjunto deles (planetas, estrelas, galáxias ou aglomerado de galáxias).

O fenômeno é acromático, ou seja, não depende do comprimento de onda. Além disso, difere das lentes na ótica convencional porque o foco não é bem definido. As imagens múltiplas, ou talvez melhor, as miragens gravitacionais, apresentam distorções, amplificações e atrasos relativos (time delay) na propagação da luz até as imagens. Essas diferenças de tempo são mensuráveis quando um processo transiente ocorre na fonte.

*Endereço de correspondência: jas.lima@iag.usp.br
Os primeiros passos da nova área foram impulsionados pela deflexão da luz observada no eclipse solar total de 1919 [1] 3]. Contudo, foi somente a partir da décadas de 60-70, que o desenvolvimento teórico e possíveis aplicações do efeito de LGs progrediram mais rapidamente.

Em 1979 (60 anos após o eclipse de 1919!), foi observada a primeira formação de uma imagem dupla de uma fonte distante. Tal resultado, teve grande impacto nas aplicações de interesse astrofísico e cosmológico [4]. Eventos observados nas últimas décadas demonstraram inequivocamente que as LGs estão entre os fenômenos mais espetaculares da natureza.

A teoria de lentes gravitacionais 1 tem sido discutida com diferentes enfoques em muitos livros textos $[5-7] \mathrm{e}$

${ }^{1} \mathrm{O}$ nome LG decorre da semelhança do fenômeno com a propagação da luz em meios materiais refringentes (vidro, água, etc.). Na aproximação pós-newtoniana da relatividade geral, mostra-se que a velocidade aparente da luz, na presença da gravitação, é menor 
artigos de revisão 8 11] (antigos e recentes). Genericamente, as LGs são separadas em 2 classes distintas: (i) macrolentes e (ii) microlentes.

As macrolentes estão subdivididas em 2 tipos: Fortes e Fracas. As lentes fortes são observadas nos aglomerados de galáxias e aparecem como arcos gigantes (giant arcs) e pequenos arcos (arclets). Em geral, provocam imagens múltiplas, o que implica na presença de uma ou mais lentes com intenso campo gravitacional. $\mathrm{O}$ caso de lentes fracas ocorre quando o campo não é forte o suficiente, tal como ocorre nas inomogeneidades da distribuição média de matéria no Universo. Seu efeito básico é uma distorção das imagens. Finalmente, quando a separação angular entre imagens múltiplas é pequena, da ordem de microsegundos de arco, uma situação típica para planetas, estrelas e quasares, falamos de microlentes. Como veremos, seu efeito apesar do nome pode ser intenso.

O artigo está organizado da seguinte forma. Na seção 2 apresentamos o desenvolvimento da área de lentes gravitacionais no que chamaremos de Idade Antiga, finalizada em 1937 com os trabalhos de Zwicky (Figura 1). O período de estagnação subsequente, de relativo silêncio na literatura, denominaremos de Idade Média (1938-1962). Na seção 3, obtemos a equação descrevendo o caso mais simples, a chamada lente pontual, e também algumas de suas consequências (anéis de Einstein, imagens duplas, etc.). Na seção 4, os principais resultados teóricos e observacionais da Idade Moderna (1963-2005) são descritos, enquanto na seção 5, narramos os contributos da Idade Contemporânea (2006-2019). Na seção 6, discutimos brevemente um desafio futuro, a conexão entre lentes e ondas gravitacionais; uma descoberta que nos projetou na era da astronomia multi-mensageira. Finalmente, na seção 7, concluímos o artigo tecendo as últimas considerações sobre as lentes, miragens gravitacionais e as perspectivas da área.

\section{Lentes Gravitacionais: Idade Antiga \& Média}

"The probability that nebulae which act as gravitational lenses will be found becomes practically a certainty"(Fritz Zwicky, 1937 ${ }^{2}$

A ideia de que a gravidade interfere na propagação da luz é uma parte razoavelmente conhecida e venerável da história da ciência. No último quartel do século XVII, Newton tornou-se o principal defensor da teoria corpuscular da luz, tanto a nível experimental quanto teórico, culminando com seu famoso livro de ótica publicado em 1704 [14]. Numa teoria corpuscular, é natural pensar que a luz, movendo-se nas proximidades de corpos celestes,

$\overline{\text { do que seu valor no vácuo }}\left(c^{\prime}=\mathrm{c} / \mathrm{n}\right)$, ou seja, o índice de refração efetivo $n$ é maior do que a unidade (ver Apêndice A).

${ }^{2}$ A probabilidade de encontrar nebulosas atuando como lentes gravitacionais torna-se praticamente uma certeza.

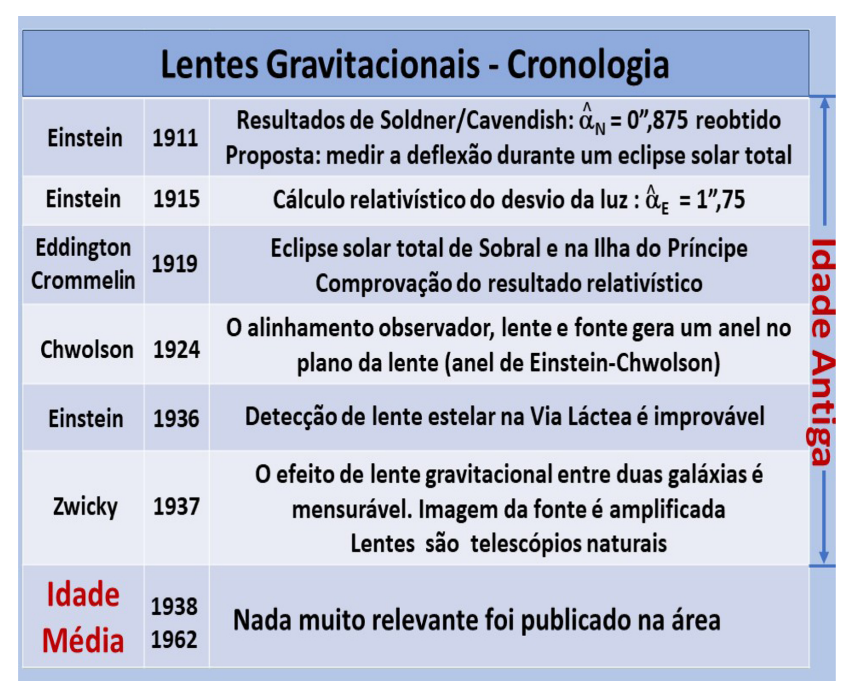

Figura 1: Lentes gravitacionais (1911-1962). Linha do tempo com os principais avanços da área de LGs na Idade Antiga. Afora o experimento crucial do eclipse de 1919 , todos os resultados da idade antiga são teóricos. Por completeza mostramos o período de estagnação (idade média). Somente após um silêncio de 25 anos, a área seria impulsionada por novas previsões teóricas e descobertas observacionais.

seja gravitacionalmente defletida, embora Newton não tenha apresentado um cálculo explícitd ${ }^{3}$

A influência da gravidade sobre o movimento da luz teve outra conexão interessante, associada aos objetos que no Século XX seriam chamados de buracos negros. John Michell (1724-1793) e Pierre Laplace (1749-1827), demonstraram que estrelas de massas suficientemente altas poderiam ser completamente escuras, ou seja, nem mesmo a luz poderia escapar da superfície 15, 16. Em outras palavras, a velocidade dos corpúsculos de luz, emitidos por um corpo esférico de massa $M$ e raio $R$, poderia ser menor do que a velocidade de escape, $v_{e}=$ $\sqrt{2 G M / R}$, na sua superfíci ${ }^{4}$.

Em 1801, Johann Soldner (1776-1833) quantificou a visão newtoniana publicando o primeiro cálculo explícito do desvio da luz nas proximidades de um corpo esférico de massa $M$ e raio $R$, supondo um corpúsculo luminoso incidindo com parâmetro de impacto $b \geq R$ [17]. No seu cálculo de espalhamento balístico para pequenos ângulos de deflexão, provocados por uma força $F \propto 1 / r^{2}$, Soldner obteve - numa notação mais moderna - a seguinte deflexão angular 5 .

\footnotetext{
${ }^{3}$ Uma visão histórica da ótica newtoniana, incluindo a herança recebida dos predecessores e sua ambivalência sobre a natureza da luz, pode ser vista em Nussenzveig 12] e Martins \& Silva [13.

${ }^{4}$ Quando a velocidade de escape é igual a velocidade da luz $\left(v_{e}=\right.$ $c)$, temos o raio gravitacional, $R=R_{g}=2 G M / c^{2}$, ou raio de Schwarzschild do corpo 18. Para o Sol $R_{g} \simeq 3 \mathrm{Km}$, mostrando que o Sol não se comporta como um buraco negro. Essa ideia seminal de buracos negros exigiria uma nova teoria de gravidade (Relatividade Geral) e quase 2 séculos seriam necessários para os "objetos invisíveis" adquirirem o presente status de corpos celestes. ${ }^{5}$ Em 1921, nos arquivos de Henry Cavendish (1731-1810), foi encontrado o mesmo resultado de Soldner, porém sem detalhes do cálculo. Cavendish fixou a velocidade do corpúsculo $v=c$ para
} 


$$
\hat{\alpha}_{N}=\frac{2 G M}{c^{2} b} \equiv 0^{\prime \prime}, 875\left(\frac{M}{M_{\odot}}\right)\left(\frac{R_{\odot}}{b}\right) .
$$

onde $G$ é a constante gravitacional de Newton, $b$ o parâmetro de impacto e $M_{\odot}=1,98 \times 10^{33} \mathrm{~g}$ e $R_{\odot}=$ $6,96 \times 10^{10} \mathrm{~cm}$ são, respectivamente, a massa e raio do Sol. Quando a luz defletida pelo Sol $\left(M=M_{\odot}\right)$, passando rasante a sua superfície $\left(b=R_{\odot}\right)$, a expressão acima fornece o resultado newtoniano, $\hat{\alpha}_{N}=0^{\prime \prime}, 875$.

Em 1905, Einstein publicou sua teoria da relatividade especial (ou restrita) [20]. A relatividade galileana e a dinâmica newtoniana foram generalizadas. O novo paradigma teórico também exigia uma modificação da teoria gravitacional de Newton. A noção de ação à distância e velocidade de propagação infinita, subjacente a visão newtoniana, era incompatível com as ideias de causalidade da relatividade restrita. De fato, os fundamentos da relatividade especial implicam que os campos físicos devem se propagar com velocidade finita. No mesmo ano, Henri Poincaré (1854-1912) também argumentou que uma descrição matemática consistente do campo gravitacional deveria prever uma velocidade finita de propagação e a existência de radiação gravitacional [21.

Em 1911, Einstein reiniciou sua busca pela teoria relativística da gravitação investigando a influência da gravidade sobre a propagação da luz com base no seu Princípio de Equivalência 22]. Como resultado, sem conhecimento prévio e de forma completamente distinta, a expressão anteriormente deduzida por Soldner foi reobtida. No entanto, diferente de Soldner, Einstein enfatizou a necessidade dos astrônomos tentarem medir o efeito durante um eclipse total do Sol. Em suas palavras: "Como as estrelas fixas das regiões do céu que são vizinhas do Sol se tornam visíveis quando ocorrem eclipses solares, esta consequência da teoria pode confrontar-se com a experiência" 23.

Em 1915, ao entender que a luz propaga-se ao longo de uma geodésica, a "linha reta"do espaço-tempo curvo, Einstein recalculou o ângulo de deflexão, $\hat{\alpha}_{E}$, obtendo:

$$
\hat{\alpha}_{E}=\frac{4 G M}{c^{2} b} \equiv 1 ", 75\left(\frac{M}{M_{\odot}}\right)\left(\frac{R_{\odot}}{b}\right) .
$$

O novo resultado difere por um fator 2 do valor newtoniano original (cf. Eq. (1)) e reflete a influência da curvatura do espaço-tempo (Figura 2), provocada por uma concentração de massa nas proximidades do caminho da luz.

Em ordem de grandeza, os dois resultados acima seguem de simples considerações dimensionais. Para um raio de luz aproximando-se de um corpo de massa $M$, existem apenas duas escalas de comprimentos naturais; o raio gravitacional (Schwarszchild) $R_{g}=2 G M / c^{2}$ (ver rodapé 4) e o parâmetro de impacto $b$. O ângulo de deflexão é adimensional, portanto, a menos de uma constante numérica $(\gamma)$, podemos escrever:

$r=\infty$, enquanto Soldner tomou $v=c$ para $r=R_{\odot}$. Segundo Will [19], as diferentes condições de contorno geram correções de segunda ordem distintas, mas os resultados coincidem em primeira ordem.

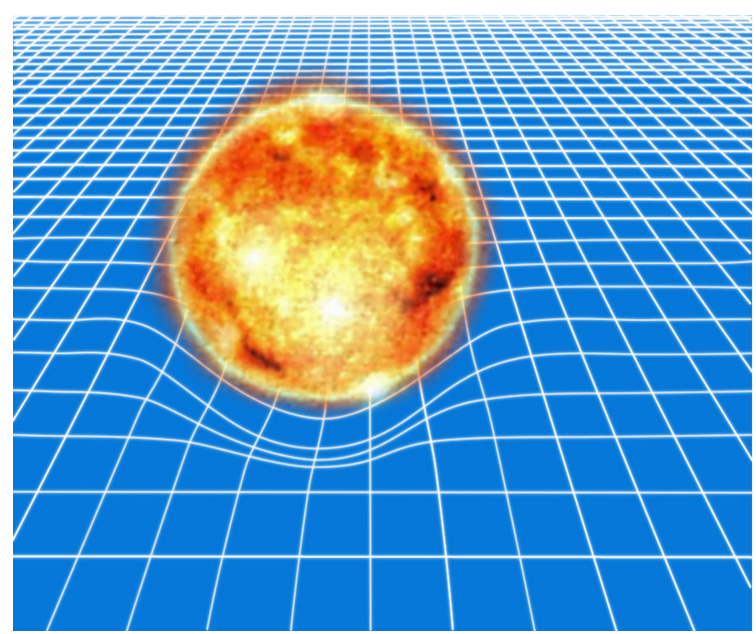

Figura 2: Geometria, curvatura e matéria. Na teoria gravitacional de Einstein, a geometria do espaço-tempo depende da presença da matéria. A curvatura pode ser visualizada pelo efeito de um corpo sobre uma folha de borracha. Longe da fonte, o efeito sobre o contínuo espaço-tempo é desprezível, enquanto nas suas proximidades torna-se curvo, alterando o movimento de todas as partículas, inclusive da luz.

$$
\hat{\alpha}=\gamma \frac{R_{g}}{b}=\gamma \frac{2 G M}{c^{2} b},
$$

onde $\gamma=1$ no caso newtoniano e $\gamma=2$ no relativístico.

A Figura 3 ilustra o efeito. Um raio de luz vindo de uma estrela distante sofre, ao passar próximo ao Sol, um desvio tal que a estrela é vista deslocada da posição real. Historicamente, a deflexão da luz pelo Sol foi o primeiro exemplo observado de uma lente gravitacional.

$\mathrm{Na}$ Figura 4, vemos como a diferença angular entre as duas posições (aparente e real) da estrela pode ser medida. O final do processo consiste em superpor as placas fotográficas (Figura 4c) imagiadas na presença do sol, durante o eclipse (Figura $\mathbf{4} \mathbf{b}$ ) e algum tempo antes (ou depois), quando o Sol, no período noturno, está fora do campo de observação (Figura $\mathbf{4 a}$ ). Numericamente, o efeito é muito pequeno, contudo, a diferença representada pelo fator 2 é crucial e quantifica, neste tipo de

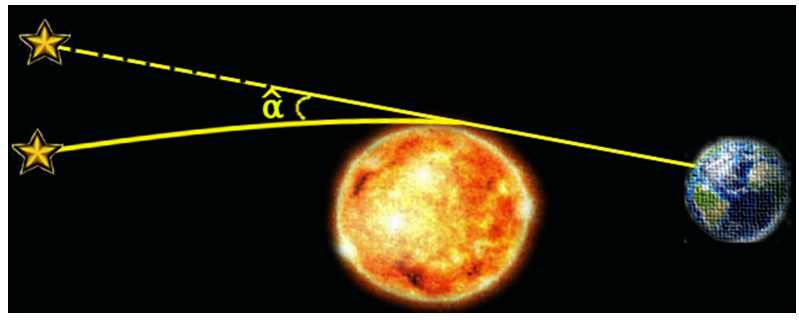

Figura 3: Deflexão da luz estelar pelo Sol. A luz emitida por uma estrela sofre uma deflexão de um ângulo $\hat{\alpha}$ ao passar próximo ao Sol. O valor do ângulo depende crucialmente da teoria de gravidade adotada no seu cálculo (Newton ou Einstein) e diferem por um fator 2 a favor da teoria de Einstein, $\hat{\alpha}_{E}=2 \hat{\alpha}_{N}$ (mais detalhes no texto). 


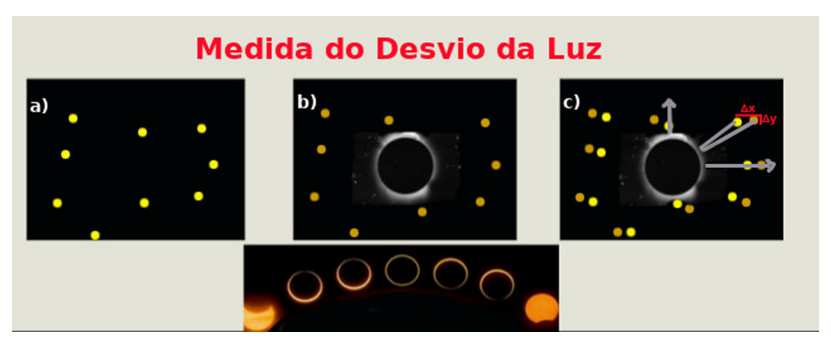

Figura 4: Medida do ângulo de deflexão em 3 etapas. a) Estrelas são imagiadas no campo de visão sem a presença do Sol. b) Posição deslocada das mesmas estrelas no momento do eclipse, e c) Superposição final das duas chapas fotográficas e determinação do ângulo de desvio. O resultado revelou-se de acordo com a teoria de Einstein.

experiência, o afastamento entre as ideias de Newton e Einstein.

É bem conhecido que a determinação deste valor durante o eclipse solar de 1919, em Sobral e na Ilha do Príncipe (costa atlântica da África) foi a segunda confirmação observacional da Relatividade Geral. A primeira tinha sido a explicação teórica do desvio anômalo do periélio de Mercúrio, um problema que estava em aberto desde 1859 [24]. No entanto, podemos dizer que era um resultado para iniciados. Por outro lado, o caráter observacional e pedagógico envolvendo a deflexão da luz, representou a base da enorme popularidade adquirida por Einstein. O experimento do encurvamento da luz pelo Sol foi repetido inúmeras vezes e todas confirmaram o resultado de Einstein 6 .

Considerando que a relação de Einstein (2) foi confirmada em 1919, existem duas consequências teóricas imediatas para o fenômeno de lentes gravitacionais: (i) a equação pode ser utilizada de forma inversa, ou seja, se o raio do objeto defletor é conhecido, a deflexão medida implica na determinação de sua massa, e (ii) a determinação da massa independe da natureza da matéria constituinte [seja matéria bariônica (nêutrons, prótons, elétrons, etc.)], ou mesmo um tipo de matéria ainda desconhecida. A partir da década de 80, tais efeitos se revelariam importantes no domínio cosmológico.

Em 1924, Chwolson [26 argumentou que no caso de alinhamento perfeito entre observador, lente e fonte, a imagem da estrela mais distante (fonte) seria vista como um anel em torno da lente, a estrela mais próxima. Esse efeito foi posteriormente denominado anel de Einstein 7

Em 1936, Einstein 27] fez o cálculo do anel e concluiu também que seria improvável a observação do encurvamente por estrelas mais próximas, da luz emitida por estrelas distantes dentro da nossa galáxia.

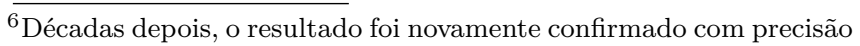
melhor que $0,02 \%$ utilizando técnicas interferométricas na faixa de rádio 25 .

${ }^{7} \mathrm{Um}$ leitor mais atento perguntaria: por que não anel de Chwolson? Em 1997, encontrou-se um caderno de anotações de Einstein com o cálculo do anel feito em 1912. Como seria esperado, o resultado continha o erro do fator 2, oriundo da deflexão obtida em 1911. O artigo de Einstein 27] é baseado nas antigas notas 28]. O nome anel de Einstein-Chwolson (ainda) não tornou-se popular.
}

Em 1937, Fritz Zwicky (1898-1974) publicou dois artigos memoráveis, ambos de meia página 29, 30]. No primeiro, ele argumentou que as nebulosas (o nome das galáxias na época), seriam mais adequadas do que estrelas para observações do fenômeno de lentes gravitacionais, o que seria até natural, pois o ângulo de deflexão é proporcional a massa. Ressaltou também o interesse da pesquisa por outras razões, dentre elas: (i) as galáxias oferecem a possibilidade de um teste adicional para a relatividade geral, (ii) o efeito de lente gravitacional seria amplificado mais de uma ordem de magnitude, quando uma galáxia inteira desempenhasse o papel de lente para outra ainda mais distante, tornando sua detecção bastante provável, e (iii) o efeito forneceria uma determinação direta da massa da galáxia lente e poderia sugerir novas linhas de investigação para os problemas cosmológicos.

Os resultados de Zwicky não tiveram influência imediata, ou seja, não produziram eco na literatura. Transcorreu um longo e silencioso período sem descobertas relevantes na área ${ }^{8}$ definindo neste artigo a Idade Média das Lentes Gravitacionais (ver Figura 1).

Os artigos seminais de Zwicky seriam retomados somente a partir dos anos 60, com a descoberta dos quasares e novas propostas teóricas. No entanto, antes de continuar nossa cronologia, apresentaremos alguns conceitos básicos da teoria de lentes gravitacionais. Como veremos a seguir, os resultados são todos de natureza puramente geométrica. Além de simples, serão úteis para entender, nas seções seguintes, os principais contributos da Idade Moderna e Contemporânea.

\section{Equação da Lente, Anel de Einstein, Imagens Duplas, Múltiplas e Magnificação}

O exemplo mais antigo e pedagógico de lenteamento gravitacional ocorre quando a lente (e a fonte) podem ser tratadas como objetos pontuais. A fonte pontual (ou lente de Schwarzschild) é um caso particular de simetria circular no plano da lente, como ocorre, por exemplo, numa galáxia elíptica. A descrição geral é válida para campos gravitacionais estacionários fracos, pequenos desvios angulares e quando o tamanho típico do defletor é muito menor do que o meio de propagação (lente fina). A lente pontual discutida a seguir é uma manifestação do que é usualmente chamado de microlente.

\subsection{Equação da Lente}

Considere uma fonte de luz $(\mathrm{F})$ bem atrás de uma lente pontual (L) de massa M. Para um observador (O), a luz defletida poderá seguir dois caminhos distintos ou no caso "geometricamente degenerado" formar um anel.

\footnotetext{
${ }^{8}$ Provavelmente, isto aconteceu devido a $2 \mathrm{a}$. guerra mundial e a concentração de grande parte da comunidade em áreas também emergentes e de interesse teórico, econômico e militar, notadamente, física nuclear, plasmas, partículas e suas aplicações.
} 
Na Figura 5 ilustramos a geometria canônica de uma microlente pontual (estática) na chamada aproximação da ótica geométrica 9 . Em geral, os ângulos são tão pequenos que as vezes não é possível resolver a fonte distante por trás da lente, embora as imagens (miragens) distorcidas sejam mais facilmente visíveis (as imagens tem separações angulares menores do que 30 segundos de arco). Os resultados a seguir permanecem válidos mesmo quando o sistema está tão distante que o modelo cosmológico padrão $(\Lambda \mathrm{CDM})$ precisa ser considerad ${ }^{10}$.

$\mathrm{O}$ ângulo $\hat{\alpha}$ é definido pelo valor de Einstein (ver Eq. (2)) e claramente depende do parâmetro de impacto $b$ ou, equivalentemente, do ângulo $\theta$

$$
\hat{\alpha}=\frac{4 G M}{c^{2} b} .
$$

Considerando que o ângulo $\beta=\theta-\alpha$ (ver Figura 5 obtemos a chamada Equação da Lente:

$$
\beta=\theta-\hat{\alpha} \frac{d_{L F}}{d_{O F}} .
$$

onde utilizamos o resultado válido para pequenos ângulos, $\alpha=\hat{\alpha} d_{L F} / d_{O F}$. Note também que o parâmetro de

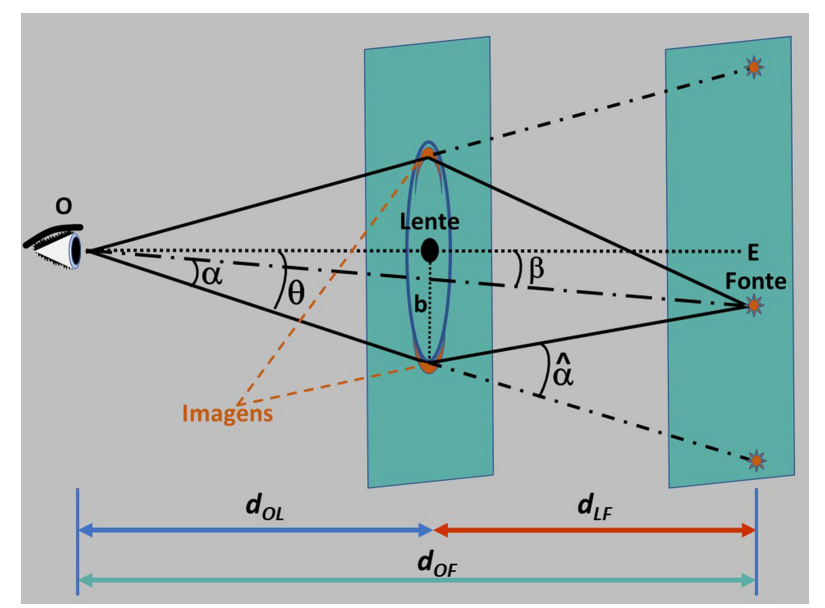

Figura 5: Geometria para eventos de lente pontual. As respectivas distâncias entre observador $(O)$, lente $(L)$ e fonte $(F)$ são $d_{O L}, d_{L F}$ e $d_{O F}$. A luz emitida pela fonte é defletida próxima a lente. Os ângulos $\alpha, \theta$ e $\beta$ são pequenos e medidos em relação ao eixo ótico (EO). Note que $\alpha$ é o ângulo entre a fonte e uma das imagens, enquanto $\beta$ e $\theta$ especificam, respectivamente, a posição da fonte no céu e da imagem inferior. O ângulo $\hat{\alpha}$ é a deflexão angular (valor de Einstein) e b o parâmetro de impacto. Para $\beta \neq 0$, duas imagens (distorcidas) são formadas, mas o caso $\beta=0$ (alinhamento total) é degenerado, com a imagem formando o chamado anel de Einstein.

\footnotetext{
${ }^{9} \mathrm{Na}$ aproximação da ótica geométrica, o comprimento de onda da radiação deve ser bem menor do que todas as escalas macroscópicas envolvidas no processo $(\lambda<<L)$.

${ }^{10} \mathrm{~A}$ única diferença é que devido a expansão universal, as distâncias envolvidas, chamadas de distâncias de diâmetro angular $\left(d_{A}\right)$, devem levar em conta a presente taxa de expansão do Universo (constante de Hubble, $\left.H_{0}\right)$, o conteúdo material $\left(\Omega_{M}+\Omega_{\Lambda}=1\right)$ e o desvio para o vermelho (redshift $z$ ) das fontes distantes 31 34].
}

impacto é dado por $b=d_{O L} \theta$. Substituindo em (3) e inserindo o resultado na expressão (4), a equação da lente pontual pode ser reescrita como:

$$
\beta=\theta-\frac{\theta_{E}^{2}}{\theta}
$$

onde definimos,

$$
\theta_{E}^{2}=\frac{d_{L F}}{d_{O F} d_{O L}} \frac{4 G M}{c^{2}} .
$$

Como veremos, o valor de $\theta_{E}$ está associado a uma escala característica fundamental na teoria de lentes.

\subsection{Anel de Einstein}

Quando observador, lente e fonte, estão alinhados no eixo ótico $\mathbf{O E}$ temos uma situação curiosa. Neste caso $\beta=0$ (ver Figura 5). As duas possíveis soluções de (6) são degeneradas, no seguinte sentido: devido a simetria rotacional, a imagem é vista como um anel, bastante referido como anel de Einstein. Sua abertura angular medida do centro da lente é dado por:

$$
\theta_{E}=\sqrt{\frac{d_{L F}}{d_{O F} d_{O L}} \frac{4 G M}{c^{2}}}=\sqrt{\frac{4 G M}{c^{2} D}} .
$$

$\mathrm{O}$ valor de $\theta_{E}$ depende de uma combinação de todas as distâncias envolvidas e não apenas da distância lentefonte. A quantidade, $D=\left(d_{O L} d_{O F}\right) / d_{L F}$, fornece a escala de distância efetiva do processo. Note também que para uma lente esférica alinhada basta considerar a distribuição de matéria (perfil de densidade), cuja massa total é $M=M\left(\theta_{E}\right)$.

Como foi visto, o anel de Einstein, $\theta_{E} \propto(M / D)^{1 / 2}$. Logo, introduzindo $M_{\odot}$ e uma distância típica (Via Láctea), $\mathrm{D}=10 \mathrm{Kpc}$, temos: $\theta_{E} \simeq\left(10^{-3}\right)^{\prime \prime}\left(\frac{M}{M_{\odot}}\right)^{1 / 2}\left(\frac{D}{10 k p c}\right)^{-1 / 2}$. Para uma galáxia elíptica de $M=10^{12} M_{\odot}$, a uma distância efetiva $D=1 G p c$, obtemos $\theta_{E} \simeq 1$ ", o que mostra ser bem mais fácil o lenteamento de galáxias.

Na Figura 6 mostramos um exemplo do anel de Einstein formado por uma galáxia elíptica. Casos mais complexos envolvendo distribuições finitas de matéria sem simetria, são também analiticamente tratáveis com as devidas integrações (na lente, fonte ou em ambos)

\subsubsection{Imagens Duplas}

A equação da lente pontual (ou circular) descrita por (6), pode ser reescrita como

$$
\theta^{2}-\beta \theta-\theta_{E}^{2}=0
$$

cujas soluções correspondem a duas imagens:

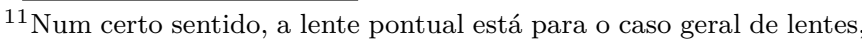
assim como o campo elétrico da carga pontual está para os campos de distribuições finitas de cargas. Ou seja, casos mais gerais podem ser obtidos por integrações (na fonte e na lente) e/ou no aumento da dimensionalidade do problema ver 5,6 .
} 


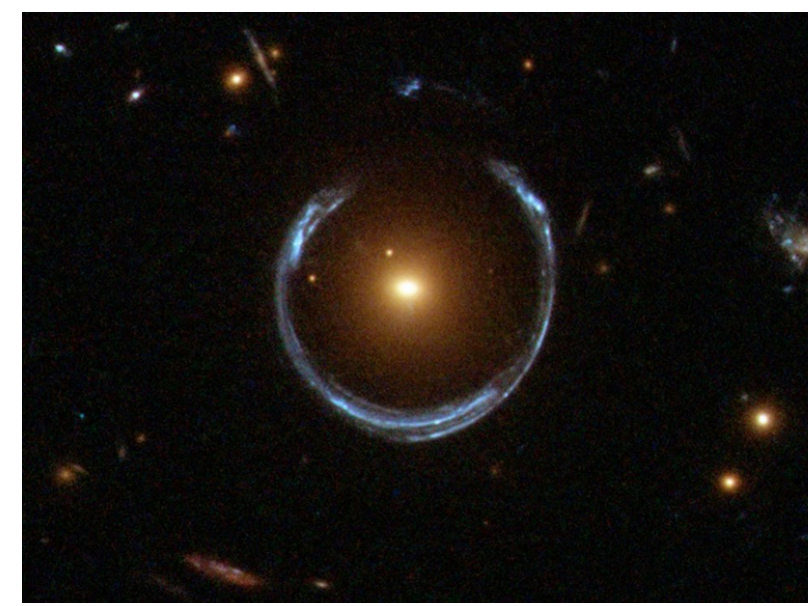

Figura 6: Formação de um anel de Einstein galáctico, como sugerido por Zwicky. A imagem de uma galáxia azul mais distante (fonte) foi distorcida por uma galáxia elíptica mais próxima (lente). Devido a precisão do alinhamento a galáxia de fundo é distorcida formando um anel quase completo (ver texto). Suas propriedades permitem determinar o conteúdo de massa da galáxia, incluindo a matéria escura (crédito da imagem: ESA/Hubble \& NASA).

$$
\theta_{ \pm}=\frac{1}{2}\left(\beta \pm \sqrt{\beta^{2}+4 \theta_{E}^{2}}\right) .
$$

Note que as duas imagens estão em lados opostos, mas não são simétricas. Das soluções acima e também da Figura 5 vemos que uma das imagens (superior) está dentro do anel de Einstein e a inferior fora. Tal assimetria é gerada pelo posicionamento da fonte (se está acima ou abaixo do eixo ótico). Se a fonte estivesse acima, seria a imagem inferior que estaria dentro do raio de Einstein $\left(\theta<\theta_{E}\right)$ e vice-versa. Portanto, uma das imagens está sempre dentro e a outra fora do anel de Einstein. Observe também que a separação entre as imagens

$$
\Delta \theta_{ \pm}=\theta_{+}-\theta_{-}=\sqrt{4 \theta_{E}^{2}+\beta^{2}} \geq 2 \theta_{E}
$$

tem seu valor mínimo determinado por $\theta_{E}$. Se $\beta$ cresce a separação aumenta.

\subsection{Magnificação}

O lenteamento gravitacional preserva o brilho superficial da fonte, pois o número de fótons emitidos é conservado. Em outras palavras, uma LG apenas redistribui os fótons emitidos pela fonte. No entanto, o ângulo sólido da fonte pode ser alterado pelo efeito de lente e, portanto, o mesmo acontece com o fluxo total. A variação do fluxo total depende da razão entre os ângulos sólidos de cada imagem e da fonte. Como resultado, podemos definir a magnificação (e o seu oposto, a demagnificação ou enfraquecimento) como uma razão entre áreas [8]:

$$
\text { Magnificação } \equiv \mathcal{M}=\frac{\text { área da Imagem }}{\text { área da Fonte }} .
$$

É possível também mostrar que a razão acima para lentes pontuais implica que a magnificação de cada imagem pode ser escrita como (ver material suplementar)

$$
\mathcal{M}_{ \pm}=\frac{\theta_{ \pm}}{2 \beta}\left(\frac{\beta}{\sqrt{\beta^{2}+4 \theta_{E}^{2}} \pm 1}\right),
$$

onde $\theta_{ \pm}$são as soluções das imagens na equação 10 Finalmente, introduzindo a quantidade normalizada, $x=$ $\beta / \theta_{E}$, segue que:

$$
\mathcal{M}_{ \pm}=\frac{x^{2}+2}{2 x \sqrt{x^{2}+4}} \pm \frac{1}{2},
$$

e somando as duas expressões acima, temos a magnificação total:

$$
\mathcal{M}=\mathcal{M}_{+}+\mathcal{M}_{-}=\frac{x^{2}+2}{x \sqrt{x^{2}+4}} .
$$

Como uma ilustração, considere uma fonte sobre o raio de Einstein, ou seja, $\beta=\theta_{E}$. Neste caso, temos $x=1 \mathrm{e}$ uma magnificação total de $\mathcal{M}=1,34$. Vários exemplos de magnificação (demagnificação) foram detectados até o presente.

\subsection{Lentes como Telescópios Naturais}

Astrônomos costumam também dizer que telescópios são máquinas do tempo, pois permitem saber como eram os objetos ou mesmo o Universo no passado. No mesmo sentido, as lentes gravitacionais funcionam como verdadeiros telescópios naturais. Isto ocorre devido ao efeito de magnificação, pois as imagens parecem mais brilhantes do que a fonte. Em muitos casos, a magnificação possibilita a detecção de uma fonte distante. Proto-galáxias em altos redshifts tem sido descobertas e seu espectro revelado por efeitos de lente forte agindo como um "telescópio"natural. Um efeito que pode também explicar porque quasares com altos redshifts podem ser encontrados próximos a galáxias de baixos redshifts.

Em 2017, foi estabelecido um novo recorde de magnificação: uma galáxia distante, foi imagiada pelo Hubble Space Telescope (HST) graças a um aumento de 30 vezes pelo efeito de lente gravitacional 35 .

\subsection{Imagens Múltiplas e Critérios na Identificação do Lenteamento}

Como foi visto, numa lente pontual ou circularmente simétrica, formam-se duas imagens ou um anel de Einstein. No caso geral, assimétrico, ou na presença de um conjunto de lentes, o tratamento matemático é mais complexo, pois a equação escalar da lente (5) torna-se vetorial (ver [5] p. 29-31, [6], p. 33). O tratamento matemático do caso geral está fora do escopo do presente artigo, embora seja simples de entender fisicamente a formação de imagens múltiplas.

Na Figura 7 mostramos uma visão qualitativa da formação de várias imagens. Quatro ou mais miragens podem ser formadas numa lente assimétrica ou constituída 


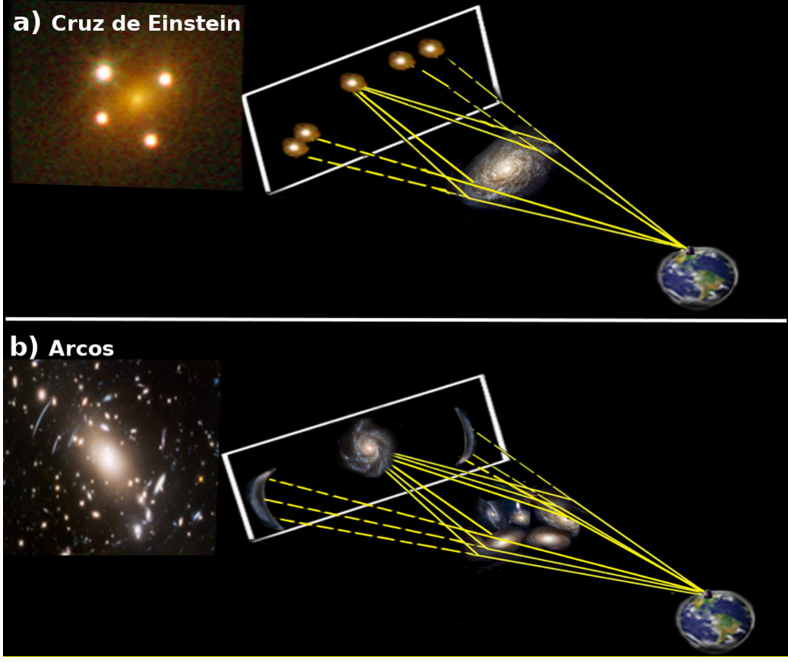

Figura 7: Imagens múltiplas. Quando existem vários caminhos óticos independentes, diferentes imagens são observadas. a) Cruz de Einstein; b) Formação de arcos (gigantes e pequenos) em aglomerados.

por muitas lentes quase pontuais. A figura é ilustrada com a chamada cruz de Einstein, um conjunto de 4 miragens ${ }^{12}$ observada Figura 7 a. No caso de muitas fontes independentes, como por exemplo, num aglomerado de galáxias, temos também formação de arcos gigantes ou de pequenos arcos (Figura 7b). As primeiras imagens de arcos gigantes (lentes fortes) foram obtidas por Lynds e Petrosian 38, enquanto os pequenos arcos foram vistos primeiramente por Tyson et al. 1990 [39]. Na seção 4.2 discutiremos brevemente lentes fortes e fracas.

Por outro lado, para os objetos observados serem classificados como imagens, alguns critérios básicos devem ser obedecidos ( 5] p. 43-44) . Os mais importantes são: (i) As 2 imagens devem estar bem próximas no céu, (ii) A razão e a forma dos fluxos nas diferentes bandas espectrais devem ser as mesmas, (iii) No caso cosmológico, as imagens devem ter o mesmo desvio para o vermelho (redshift), (iv) Uma possível lente deve estar na vizinhança das imagens, e (v) as variações temporais nas diferentes imagens (devido a variações na fonte) devem estar correlacionadas (time-delay). Retornemos agora a nossa cronologia das lentes gravitacionais.

\section{Idade Moderna}

Em 1963 foram descobertos os quasares ${ }^{13}$, uma classe de objetos com aparência quase estelar (quasistellar object, QSO no acrônimo inglês). Os quasares [40] são extremamente compactos, luminosos e estão localizados a grandes distâncias, ou seja, em redshifts mais altos do

\footnotetext{
12 Um teorema devido a William Burke (1941-1996), baseado no estudo da formação de caústicas e linhas críticas do lenteamento, demonstrou que o número de imagens múltiplas é sempre ímpar 36]; embora uma das imagens sendo demagnificada não seja vista, tal como ocorre na cruz de Einstein (ver também 37]).

13 https://pt.wikipedia.org/wiki/Quasar, consultado em 15/07/2019.
}

que a maioria das galáxias. Imediatamente percebeu-se que os QSOs formam uma população especial de fontes pontuais distantes, convenientes para formação de imagens (ou miragens) como sugerido por Zwicky. Sua luz certamente seria lenteada pelas galáxias e aglomerados na longa jornada até a Terra. A identificação dos quasare $\sqrt{14}$ revelou-se fundamental no estudo das lentes gravitacionais e demarcarão nesse texto o prelúdio da era moderna (ver Figura 8).

Em 1964, Refsdal [41,42 analisou o lenteamento por uma galáxia supondo que a fonte pontual de luz era uma supernova (SN) distante. Sendo a SN um evento transiente, a luz desviada por uma lente galáctica (situada entre a SN e a Terra), permitiria que a explosão fosse vista em momentos distintos nas diferentes imagens 15 Refsdal calculou o atraso de tempo ("time delay"), devido a diferença de percurso entre a fonte e as imagens. Isso ocorreria não apenas com SNs, mas também com qualquer fonte pulsante ou variável. Foi também argumentado que além de uma estimativa da massa da galáxia defletora, as miragens formadas determinariam a constante de Hubble - $H_{0}$ - combinando os parâmetros observados no sistema lente-fonte e no atraso de tempo. Na idade contemporânea, veremos como essa notável previsão de Refsdal poderá resolver a tensão existente nas medidas da constante de Hubble $\left(H_{0}\right)$ no contexto cosmológico (seção 5.5).

\begin{tabular}{|c|c|c|c|}
\hline \multicolumn{3}{|c|}{ Lentes Gravitacionais - Cronologia } & \\
\hline Quasar & 1963 & Descoberta dos Quasares (QSOs) & \\
\hline Refsdal & 1964 & Supernova Fonte - Atraso de tempo - Medida de $\mathrm{H}_{0}$ & \\
\hline $\begin{array}{c}\text { Walsh } \\
\text { Carswell } \\
\text { Weymann }\end{array}$ & 1979 & $\begin{array}{l}\text { Detecção de } 2 \text { Imagens do Quasar QSO 0957+561 (A,B) } \\
\text { CCDs Substituem Placas fotográficas - Operação } \\
\text { do VLA (Very Large Array) - Imagens em Rádio }\end{array}$ & $\frac{2}{2}$ \\
\hline Pacynski & 1986 & $\begin{array}{l}\text { Microlentes - Curvas de Luz - Busca de Objetos } \\
\text { Compactos (Escuros) na Via Láctea (MACHOS) }\end{array}$ & \\
\hline $\begin{array}{l}\text { Lynds } \\
\text { Petrosian }\end{array}$ & $\begin{array}{l}1986 \\
1988\end{array}$ & $\begin{array}{c}\text { Detecção de Arcos Luminosos em Aglomerados de } \\
\text { Galáxias - Imagens Distorcidas e Amplificadas } \\
\text { das Galáxias Distantes }\end{array}$ & $\frac{2}{2}$ \\
\hline Herwitt et al. & 1988 & Primeiro anel de Einstein: QSO MG1131 + 0456 & \\
\hline Bond et al. & 2004 & $\begin{array}{l}\text { Descoberta do Primeiro Exoplaneta com Lentes } \\
\text { Gravitacionais (Microlentes) }\end{array}$ & \\
\hline
\end{tabular}

Figura 8: Lentes gravitacionais na Idade Moderna (1963-2005). Linha do tempo mostrando os principais avanços teóricos e observacionais na área de lentes gravitacionais no período. A identificação dos quasares (1963) e a primeira observação de uma imagem dupla em 1979 (QSO 0957+561 A \& B) estão entre as mais extraordinárias descobertas do período.

\footnotetext{
${ }^{14}$ QSOs estão entre os eventos mais energéticos do Universo. São capazes de emitir centenas ou milhares de vezes a energia eletromagnética de nossa galáxia. Sua aparência e potência são explicadas por um superburaco negro acretando matéria da vizinhança. Atualmente, já foram observados QSOs com redshifts $z>6$.

${ }^{15}$ A partir de 1998, a experiência adquirida na busca de SNs em altos redshifts no contexto cosmológico, criou as condições adequadas para o uso de SN como fonte no processo de lente. O lenteamento de uma SN foi finalmente observado em 2014, com a descoberta da chamada Supernova de Refsdal contendo várias imagens (ver Figura 18.
} 
Em 1979, Walsh, Carswell \& Weymann 43] observaram a primeira formação de duas imagens por uma fonte extragaláctica, o quasar QSO $0957+561(A \& B)$ \&3]. Embora previamente selecionado de um catálogo de rádio fontes, as duas imagens do quasar também aparecem no ótico. A similaridade dos espectros extraído das imagens no redshift $z \simeq 1,4$, mostram que são miragens de um único objeto lenteado (Figura 9 .

$\mathrm{Na}$ Figura 10 mostramos uma imagem mais recente dos gêmeos QSO 0957+561 (A \& B), incluindo sua galáxia lente (YGKOW G1), situada no redshift $z \approx 0,36$. As imagens são os pontos mais brilhantes, separados por 5,7 segundos de arco. A lente situa-se entre os dois, estando mais próxima de B. Diversos estudos posteriores mostraram que os critérios gerais para identificação de imagens, enumerados no final da seção 3.3, são obedecidos, a menos do último pois a fonte não é variável. Provavelmente, o quasar QSO 0957+561 (e suas miragens), é a fonte mais estudada da astronomia moderna.

Naturalmente, a descoberta do quasar duplo atraiu atenção geral. Além de estimular as pesquisas em LGs, mostrou o interesse do imagiamento de objetos brilhantes e longínquos do Universo, tais como os quasares, supernovas e as primeiras galáxias. O sucesso foi também possível devido a uma profunda mudança tecnológica: a substituição das antigas placas fotográficas por CCDs, produzindo um aumento considerável de sensibilidade na detecção de fótons ${ }^{16}$

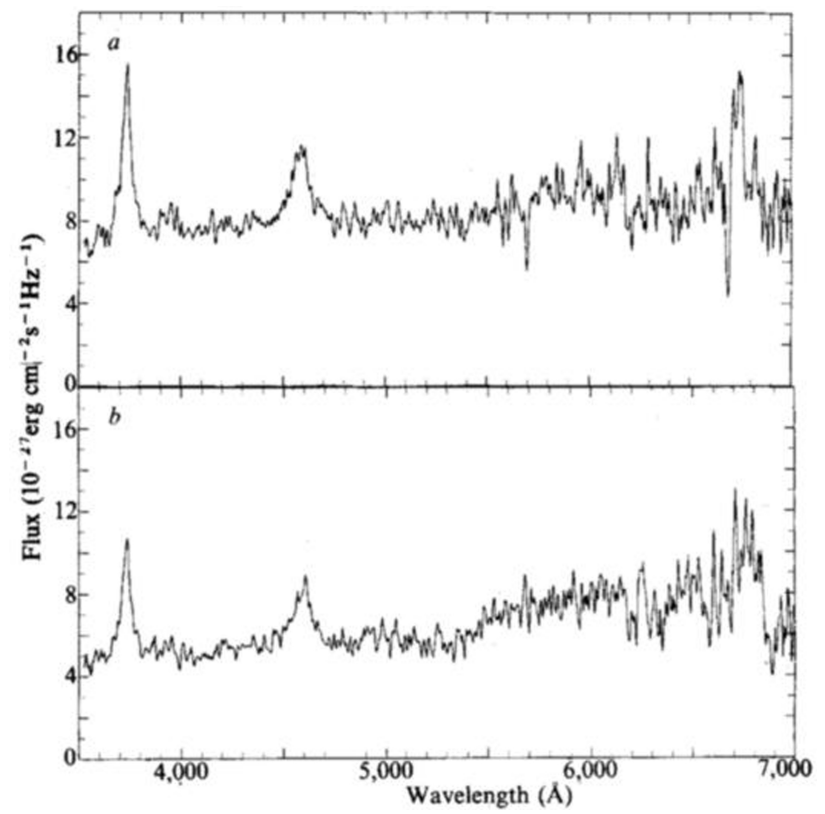

Figura 9: Espectros dos QSOs $0957+561, A$ e $B$ [43]. Note a semelhança dos espectros extraídos no mesmo redshift. Espectros mais precisos em várias bandas, revelaram que os critérios gerais para identificação de miragens, formulados no final da seção 3.3, são obedecidos nos quasares gêmeos.

\footnotetext{
${ }^{16} \mathrm{Em} 1979$, a entrada em operação do rádio interferômetro VLA (Very large Array), mostrou que os dois quasares são fontes de rádio
}

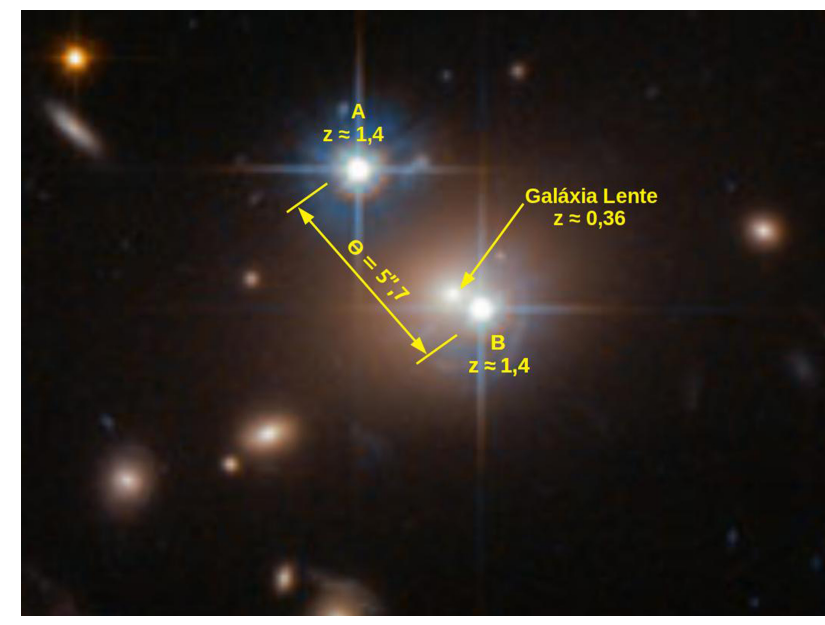

Figura 10: Imagem do telescópio espacial Hubble (HST), mostrando a dupla miragem do quasar QSO $0957+561$ (os 2 pontos mais brilhantes, $A$ e B). A galáxia lente (YGKOW G1), está mais próxima de $B$ (mais detalhes no texto). Crédito da Imagem: ESA/Hubble \& NASA (com adaptação pedagógica).

Neste ponto é importante também mencionar que o primeiro anel de Einstein, a fonte de rádio MG1131 + 0456, foi descoberto em 1988 por Hewitt e colaboradores [44], utilizando também o VLA (Figura 8).

\subsection{Lentes Gravitacionais e Matéria Escura}

Supondo que a matéria dominante é bariônica ou norma 17 a chamada curva de rotação das galáxias revelaram um paradoxo inesperado.

Para entender o mistério, considere um objeto girante de massa $m$ a uma distância $r$ do centro de uma galáxia aproximadamente esferoidal. A massa da Galáxia é $M(r)$ e a força gravitacional sobre o objeto girante é a força centrípeta $\left(F_{G}=F_{C}\right)$. Newtoniamente, a velocidade do objeto é dada por $v(r)=\sqrt{G M(r) / r}$.

Fazendo observações além da parte luminosa, teríamos $M(r)=$ constante $\Rightarrow v \propto r^{-\frac{1}{2}}$, ou seja, a velocidade tangencial obedeceria uma lei kepleriana, tal como ocorre com os planetas no caso do Sol.

No entanto, as observações mostraram que as velocidades na periferia não diminuíam, ficando aproximadamente constante, diferente do previsto newtoniamente. Uma solução possível é a existência de uma forma de matéria invísivel, ou seja, que interage gravitacionalmente, mas não brilha. Se $M(r) \propto r$, teremos $v \simeq$ const., resolvendo o problema (ver Figura 11).

No início dos anos 70, Kenneth Freeman [46], Vera Rubin e colaboradores 47, 49], sugeriram que a existência da matéria escura fria (cold dark matter, CDM no acrônimo inglês) seria a explicação mais simples para as curvas de rotação das galáxias espirais. Ostriker e

compactas de mesmo espectro. Em 1980, o uso do VLA também permitiu a identificação da galáxia lente 45. (ver Figura 10.

${ }^{17}$ A matéria existente em planetas, estrelas, etc. Tecnicamente, são partículas (pesadas) do modelo padrão da física de partículas elementares. 


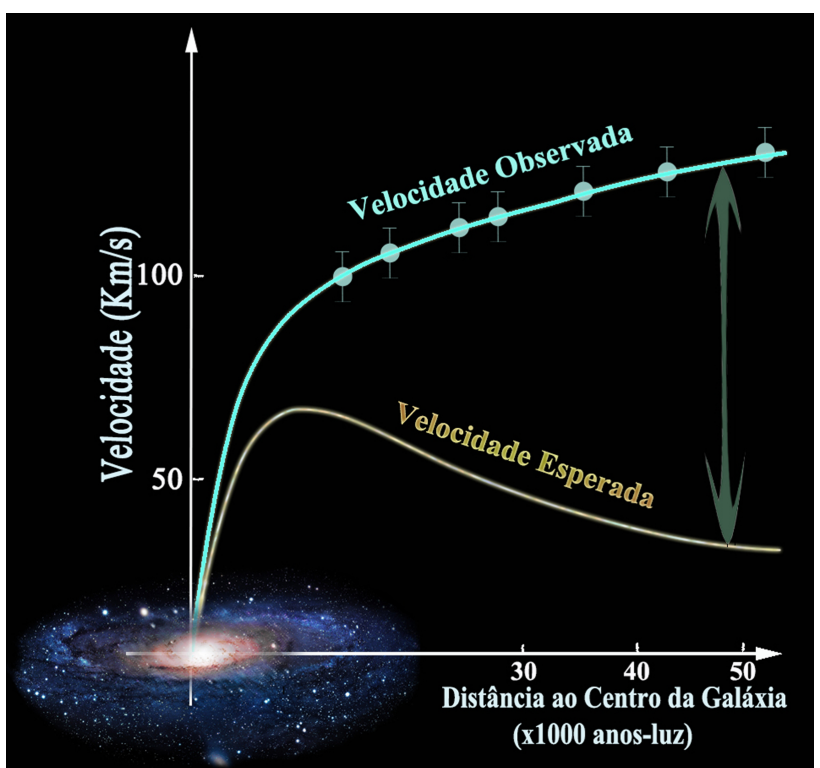

Figura 11: Curva de rotação de uma galáxia espiral. A diferença entre a curva superior (onde estão os dados) e a inferior teórica pode ser explicada pela presença de uma componente invisível que interage apenas gravitacionalmente (ver texto) .

Peebles 50 já haviam argumentado que um halo esférico de matéria escura nas galáxias espirais poderia também garantir a estabilidade do disco luminoso.

A conexão matéria escura, lentes gravitacionais e cosmologia foi imediata. Dois tipos de candidatos foram propostos: (i) objetos compactos (buracos negros, estrelas de nêutrons, anãs marrons, planetas) e (ii) um zoológico de partículas elementares estáveis, fósseis do Universo primitivo, tais como, o Higgs, Axions, Neutralinos e WIMPs ${ }^{18}$. As observações revelariam sua abundância cósmica, natureza, ou descartaria candidatos. Estava aberta a era da chamada "astronomia da matéria invisível", uma inesperada oportunidade para as LGs. Veremos a seguir como as LGs descartaram a proposta de objetos compactos como formadores dos halos escuro 19

Em 1986, Bohdan Paczynski (1940-2007), sugeriu que a variação da magnificação devido a efeitos cinemáticos em microlentes 20 poderiam identificar objetos compactos escuros no halo da Via Láctea (lentes), pois seriam iluminados por estrelas das nuvens de Magalhães 53 54 . Os objetos compactos cruzando a linha de visada das estrelas provocaria uma variação temporária do brilho (aumento seguido de um decaimento na magnificação) que poderia ser monitorado.

Várias colaborações/projetos foram organizados com esses objetivos, tais como, MACHOs ${ }^{21}$ EROS $^{22}$ e OGLE ${ }^{23}$

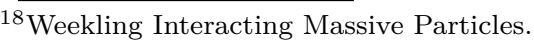

${ }^{19}$ A natureza do possível candidato a matéria escura, oriundo da física de partículas, ainda permanece indeterminada 51.52$]$

${ }^{20}$ Paczynski foi o primeiro a utilizar o termo microlente (ver 9. 453).

${ }^{21}$ MACHOs $\equiv$ Massive Compact Halo Objects

${ }^{22} \mathrm{EROS} \equiv$ Expérience pour la Recherche d'Objets Sombres

${ }^{23}$ OGLE $\equiv$ Optical Gravitational Lensing Experiment
}

A variação da magnificação com o tempo é medida pela "curva de luz"de Paczynski (Figura 12), e sua observação permite estimar a massa da lente. Como funciona o método?

A duração do evento é fixada pelo tempo característico de Einstein. Supondo uma velocidade transversa da lente constante temos, $t_{E}=r_{E} / V_{\perp}=d_{O F} \theta_{E} / V_{\perp}$, onde $r_{E}$ é o raio do anel de Einstein que depende da massa da lente e $V_{\perp}$ é a componente da velocidade perpendicular a linha de visada observador-fonte. A variação do ângulo, implica que a quantidade definindo a magnitude $x(t)=\beta(t) / \theta_{E}$ (ver seção 3.2 e 3.3) é uma função do tempo que depende da massa da lente através do anel de Einstein (ver Eq. 8 .

Todas as quantidades desconhecidas, incluindo $V_{\perp}$ podem ser estimadas combinando medidas cinemáticas e óticas com a variação máxima da curva de luz.

O método da curva de luz pode ser aplicado a 2 estrelas ou um par planeta-estrela, onde uma das estrelas ou planeta é a lente em movimento.

Em linhas gerais, a conclusão final dos projetos MACHOs e EROS com relação a possibilidade de descrição da matéria escura revelou-se negativa: Objetos compactos com massas $m \leq 30 M_{\odot}$ não são suficientemente abundantes para formar halos escuros, ou seja, não explicam as curvas de rotação.

Sua abundância cósmica inferida do halo da Via Láctea seria inferior a 20-25\%. Uma análise posterior (2003) obteve uma contribuição dos MACHOs para a matéria escura $<5 \%$ 56.

Se os objetos compactos - MACHOS - não estavam na periferia da galáxia onde estariam? Talvez no Bojo, uma região de maior densidade estelar. Na verdade, o projeto OGLE concentrou-se na região mais interna da Via Láctea, onde os efeitos de microlentes seriam mais abundantes. Os dados analisados a partir de 2003, mostrariam

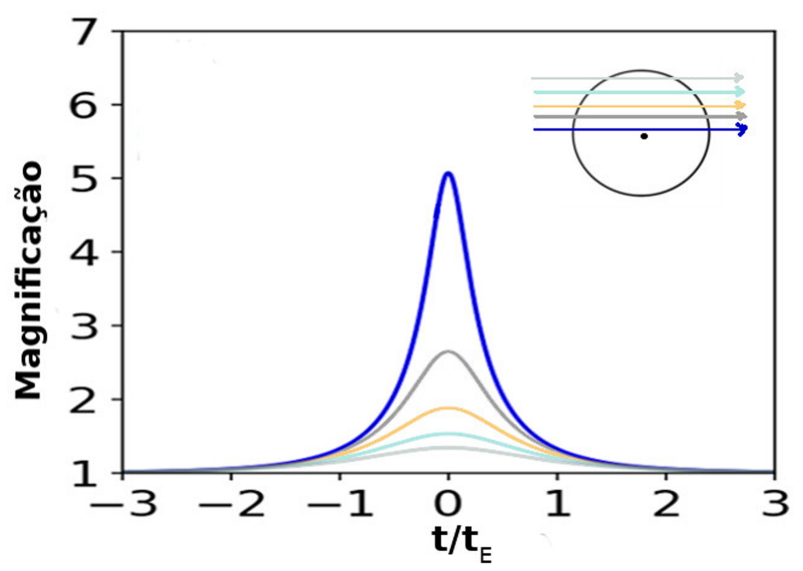

Figura 12: Variação da magnificação (curva de luz) como função do tempo em lentes pontuais. As curvas foram traçadas para 5 parâmetros de impacto diferentes (não mostrados). O circulo é o anel de Einstein e as setas indicam as trajetórias. Quanto menor o parâmetro de impacto, maior a magnificação. Note a simetria da curva em torno de $\mathrm{t}=0$. (Adaptado de $[57]$ ). 
que o OGLE teria mais sucesso na busca dos eventos de microlentes. Em particular, na busca de exoplanetas; uma possibilidade sugerida por Mao e Paczynski [57,58].

Os desenvolvimentos técnicos posteriores do OGLE e dos novos projetos na área como, MOA ${ }^{24}$ MICROFun ${ }^{25}$ e PLANET ${ }^{26}$, permitiriam a descoberta de exoplanetas a partir de 2004 .

O método é relativamente simples. Quando observamos a curva de luz de uma fonte distante cuja lente em movimento relativo é uma estrela circulada por um (ou mais planetas), pequenas anomalias ("lombadas") podem ser observadas na curva de luz.

Na Figura 13 mostramos um exemplo concreto, imagiado pela colaboração OGLE em $2006^{27}$

O conjunto de dados em tempo real consiste de 650 pontos do PLANET Danish (ESO La Silla, ponto vermelhos), PLANET Perth (azul), PLANET Canopus (Hobart, ciano), RoboNet Faulkes North (Havaí, verde), OGLE (Las Campanas, preto) e MOA (Observatório do Monte John, castanho). No canto superior direito vemos o "zoom" da anomalia planetária, cobrindo um intervalo de tempo de 1,5 dias. A curva sólida é o melhor modelo de lente binária incluindo um planeta de massa, $M_{p} \simeq 5,5 M_{\text {terra }}$, localizado a uma distância $\simeq 2,6 U A$ da estrela.

Atualmente, mais de 60 exoplanetas já foram descobertos por esta técnica, nascida dos contributos seminais de Paczynski e colaboradores (1986-1991).

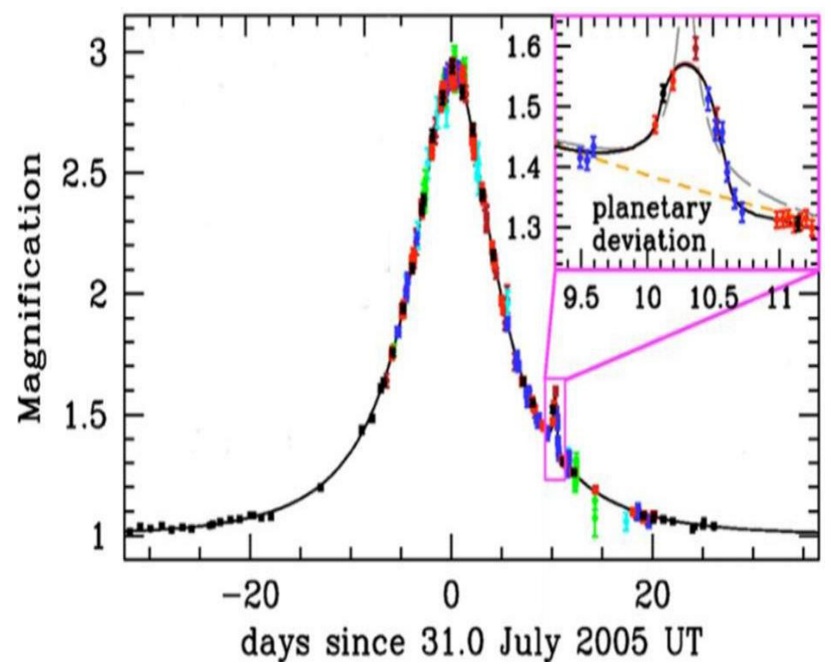

Figura 13: Curva de luz de um efeito de microlente planetária $O G L E-2005-B L G-390$. As diferentes cores representam as contribuições de vários telescópios. O canto superior é um "zoom" da anomalia planetária, cujo melhor ajuste é indicado pela linha preta sólida. O planeta tem uma massa de $\simeq 5,5 M_{\text {terra }}$ (ver texto) e está a uma distância de 2,6 UA da estrela hospedeira (extraída de [55]).

\footnotetext{
${ }^{24} \mathrm{MOA} \equiv$ Microlensing Observations in Astrophysics

${ }^{25}$ MICROFun $\equiv$ Microlensing Follow-Up Network

${ }^{26}$ PLANET $\equiv$ Probing Lens Anomaly NETwork

${ }^{27} \mathrm{O}$ primeiro evento planetário de microlentes foi obtido em 2004, com massa $M_{p}=1,5 M_{J}$ e um raio orbital de $\simeq 3 \mathrm{UA} 59$.
}

\subsection{Lentes Fortes e Fracas}

Aglomerados de galáxias são as maiores estruturas do Universo gravitacionalmente ligadas que podem estar em equilíbrio hidrostático (virializadas). Aglomerados ricos chegam a ter mais de $10^{3}$ galáxias, massas no intervalo $10^{14} M_{\odot} \leq M \leq 10^{16} M_{\odot}$ e diâmetros entre 1-2 Mpc. A composição inclui a massa estelar nas galáxias, um gás quente (plasma) no meio intergalático e matéria escura. Tal como as galáxias, os aglomerados deformam o espaçotempo nas partes internas e na vizinhança, encurvando a luz das fontes distantes. Nas regiões internas, a densidade é alta e pode defletir os feixes da frente de onda, produzindo imagens múltiplas.

Na Figura 14 (ver também 7b), vemos que as galáxias multiplicam o efeito de lentes formando extraordinários arcos, cuja análise espectroscópica revela o mesmo redshift e, portanto, são lentes de um mesmo objeto distante. Os arcos gigantes aparecem curvados na direção da região mais densa, o centro do aglomerado. Além disso, vemos da figura que sua extensão tangencial (relativa ao centro do aglomerado) é bem maior do que a largura radial. A formação de arcos gigantes e pequenos, descoberto nos anos 90 (ver também seção 3.5), caracteriza o regime de lentes fortes em aglomerados.

No limite que as deflexões causam pequenas modificações nas propriedades do objeto (brilho, posição, tamanho e forma), mas não fenômenos visualmente impressionantes, como múltiplas imagens ou arcos, o processo é chamado de lente fraca. Em geral são induzidas pelas flutuações na densidade média do Universo. As distorções observadas na periferia dos aglomerados são uma combinação homogênea de forma (convergência) com o cisalhamento ("shear"), provocados pelo efeito de lente fraca ao longo da linha de visada 8 .

Na Figura 15 mostramos o efeito qualitativo de lentes forte e fraca. No primeiro caso a luz passa mais distante

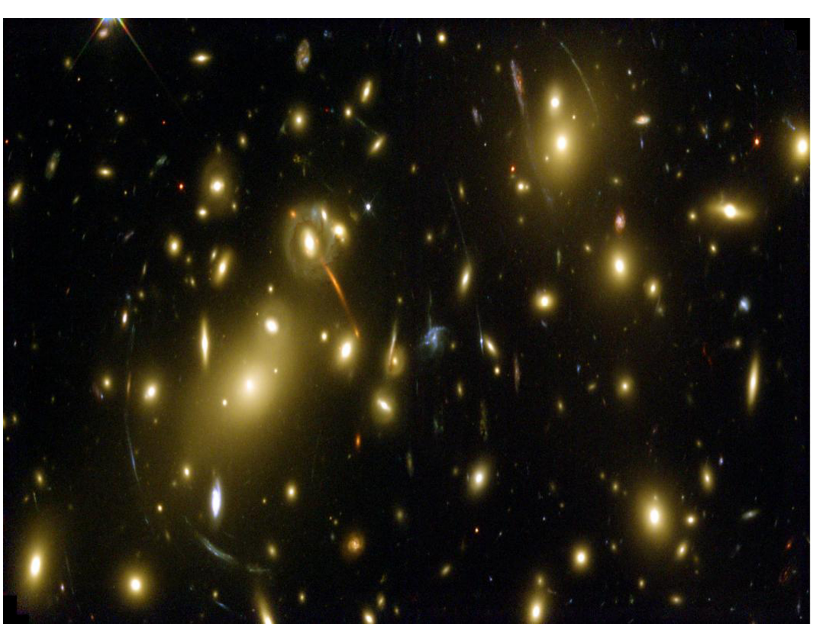

Figura 14: Imagens múltiplas, arcos gigantes e pequenos arcos. Todas as imagens são vistas no grande aglomerado Abell 2218, no redshift $z=0,175$, um dos mais espetaculares sistemas de arcos variados (Crédito da imagem: Space Telescope Science Institute). 


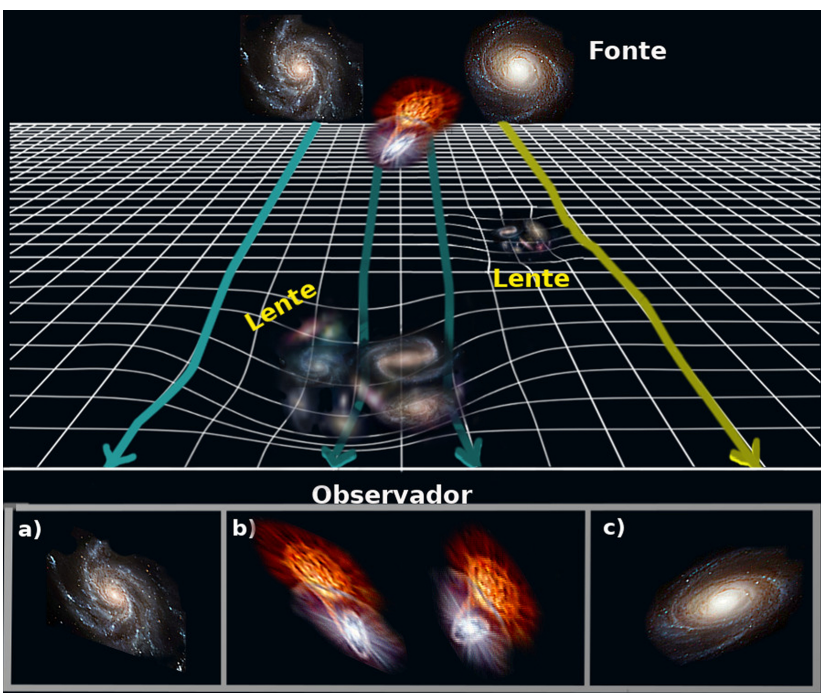

Figura 15: No caso dos exemplos (a) e (c) temos imagens distorcidas devido ao efeito de lente fraca, já no (b) temos a formação de múltiplas imagens devido ao efeito de lente forte.

do núcleo do aglomerado e o efeito pode ser uma mera distorção, um cisalhamento ("shear") na imagem; um círculo aparece como uma elipse [inserções (a) e (c)]. No caso de lentes fortes, a luz das fontes lenteadas passam na parte mais interna dos aglomerados sofrendo distorção, amplificação e multiplicação de imagens [inserção (b)].

Lente gravitacional por aglomerados de galáxias é uma das principais ferramentas cosmológicas para acessar a matéria escura e o Universo distante. Assinaturas de lentes fortes funcionam como sondas da distribuição da matéria mais interna dos aglomerados de galáxias, permitindo a localização do núcleo dos clusteres e a determinação de sua massa. A ampliação de fluxo aparente transforma aglomerados de galáxias em telescópios gravitacionais, que podem ser usados para estudar galáxias em altos redshifts que seriam, caso contrário, muito fracas para serem observadas.

O estado dinâmico do cluster e a presença de substruturas pode também ser levantada via lentes fracas. Medidas de massa com lentes fracas e de raios-X, e a velocidade de dispersão podem ser comparadas com simulações testando a estrutura da "teia cósmica"e também saber o aglomerado em equilíbrio ou se as diferentes componentes estão interagindo 6062 .

\section{Idade Contemporânea (2006-2019)}

Em 1933, Zwicky já havia inferido a existência de matéria escura, estimando a dispersão de velocidade das galáxias na região central dos aglomerados 63. Na idade moderna sua conjectura foi adotada devido a dois efeitos: (i) as curvas de rotação (seção 4.1) e, (ii) existência dos arcos como um efeito de lentes nos aglomerados.

Mais recentemente (2006), a existência da matéria escura foi também sugerida pela primeira fusão observada de dois aglomerados; um evento que ficaria conhecido como aglomerado bala ("Bullet Cluster"). As LGs desempenharam um papel crucial nessa descoberta (Figura 16), considerada até o presente a evidência mais direta da matéria escura 64,65.

\subsection{O Aglomerado Bala}

Um caso especial de mapeamento do perfil de densidade e massa total por lentes ocorre na fusão de 2 aglomerados de galáxias. O primeiro e mais popular exemplo, é o aglomerado bala, 1E 0657-56, considerado o evento mais energético conhecido no Universo desde o Big Bang ${ }^{28}$ Seu mapeamento é uma combinação de duas técnicas: raios-X e lentes gravitacionais.

$\mathrm{Na}$ Figura 16, vemos o resultado simulado do processo de fusão dos dois aglomerados de galáxias.

O plasma quente (avermelhado) foi detectado pelo observatório Chandra 29 e contém a maior parte da matéria normal ou bariônica dos dois aglomerados [66]. O perfil em forma de bala à direita é o gás do aglomerado menor e mais veloz, atravessando o gás quente do maior aglomerado durante a colisão. As áreas azuis assinalam onde as lentes gravitacionais encontraram a maior parte da massa dos aglomerados (note que o centro de gravidade não coincide com o da massa bariônica). Devido a interação, o movimento do gás defasou-se em relação ao da matéria escura, uma componente que interage apenas gravitacionalmente.

Dizendo de outra forma, o plasma de cada cluster foi retardado pela força média (mútua), de origem viscosa,

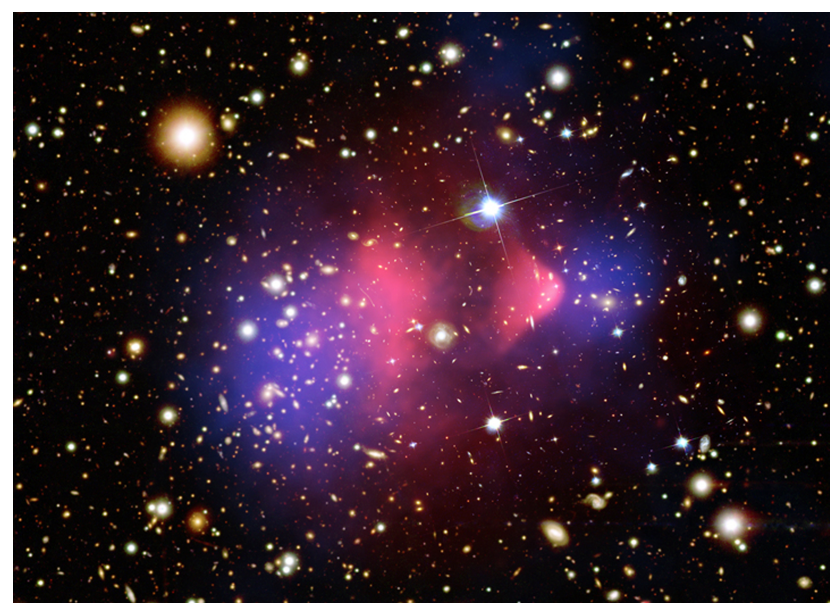

Figura 16: Aglomerado de galáxias $1 E 0657$ - 56. Devido ao perfil ficou popularmente conhecido como aglomerado bala (bullet Cluster). $\mathrm{O}$ menor cluster à direita atravessa o maior à esquerda. Nessa imagem, dados de raios-X (parte avermelhada) e o mapeamento da massa obtido de lentes fracas (azul) são sobrepostos a imagem óptica do HST (detalhes no texto). Crédito: Raios- $X$ : NASA/CXC/CfA/; Mapa de Lentes: NASA/STScl; ESO WFI; Magellan/U.Arizona/; ótico: NASA/STScl; MagelIan/U.Arizona.

\footnotetext{
${ }^{28}$ Uma simulação mostrando a formação do Bullet Cluster pode ser vista no site https://www.youtube.com/watch?v=eC5LwjsgI4I ${ }^{29}$ NASA X-Ray Observatory
} 
semelhante à resistência do líquido quando atravessado por uma bala. Durante a colisão, a matéria escura (não interagente) dos dois aglomerados, moveu-se à frente do gás quente, produzindo a separação do material escuro e bariônico vista na imagem. O resultado mostra que a matéria escura é necessária na escala dos aglomerados e reafirma seu status de componente material (não relativística) dominante no Universq ${ }^{30} 68$.

\subsection{Universo Acelerado e Lentes Gravitacionais}

A descoberta da aceleração do Universo em 1998, através das observações de Supernovas do tipo Ia, estabeleceu um novo paradigma na Cosmologia 69,70. Em 2006, o modelo acelerado, plano, composto por bárions $(\sim 5 \%)$, matéria escura $(\sim 25 \%)$ e energia escura $(\sim 70 \%)$, já era considerado o modelo cosmológico padrão. Mas não era conhecida ainda a natureza da energia escura e da matéria escura, o chamado setor cosmológico escuro.

Em 2006, o projeto Dark Energy Task Force (DETF) foi criado com o objetivo de determinar a natureza da Energia Escura [71]. A questão era decidir se o agente acelerando o Universo era a constante cosmológica $(\Lambda)$, associada a densidade de energia do vácuo, ou algum outro tipo de campo. Várias abordagens são possíves. A metodologia adotada foi a combinação de 4 diferentes subáreas (i) Oscilações Acústicas dos Bárions, (ii) Aglomerados de Galáxias, (iii) Supernovas, e (iv) Lentes Gravitacionais. Algumas abordagens recentes e resultados envolvendo a área de lentes gravitacionais serão descritas a seguir.

\subsection{SNe Ia e Lentes Fracas}

As SNe Ia são também lenteáveis pela estrutura do Universo, magnificando (ou demagnificando) seu brilho, dependendo se a linha de visada para o SNe Ia passa por regiões de sobredensidades (subdensidades) de matéria. Com suficiente número de objetos e uma modelagem adequada das lentes fracas 72,73$]$, os dados poderiam ser utilizados para estimar parâmetros cosmológicos usando a distribuição de SNe Ia (Figura 17) cruzada com a densidade observada ao longo da linha de visada, ou mesmo usando sua amplificação (à "la Paczynsky") para avaliar as propriedades do halo da matéria escura dos aglomerados 74, 75.

Em 2014, a magnificação das SNe Ia foi marginalmente detectada por Smith et al. 76. Os autores correlacionaram as magnitudes de SN Ia da amostra SDSS ${ }^{31}$ com a densidade observada ao longo da linha de visada. No entanto, a distribuição é sensível à estrutura de grande e pequena escala que é bem mais difícil de modelar.

Os dados para $z>1$, ou seja, quando o universo ainda estava desacelerado, podem ser obtidos medindose os redshifts da galáxia hospedeira. A princípio, tal

\footnotetext{
30 A existência do evento bullet cluster contradiz as teorias de gravidade que alteram a lei de força newtoniana 67], coletivamente denominadas do tipo MOND (Modified Newtonian Dynamics). ${ }^{31}$ SDSS $\equiv$ Sloan Digital Sky Survey
}

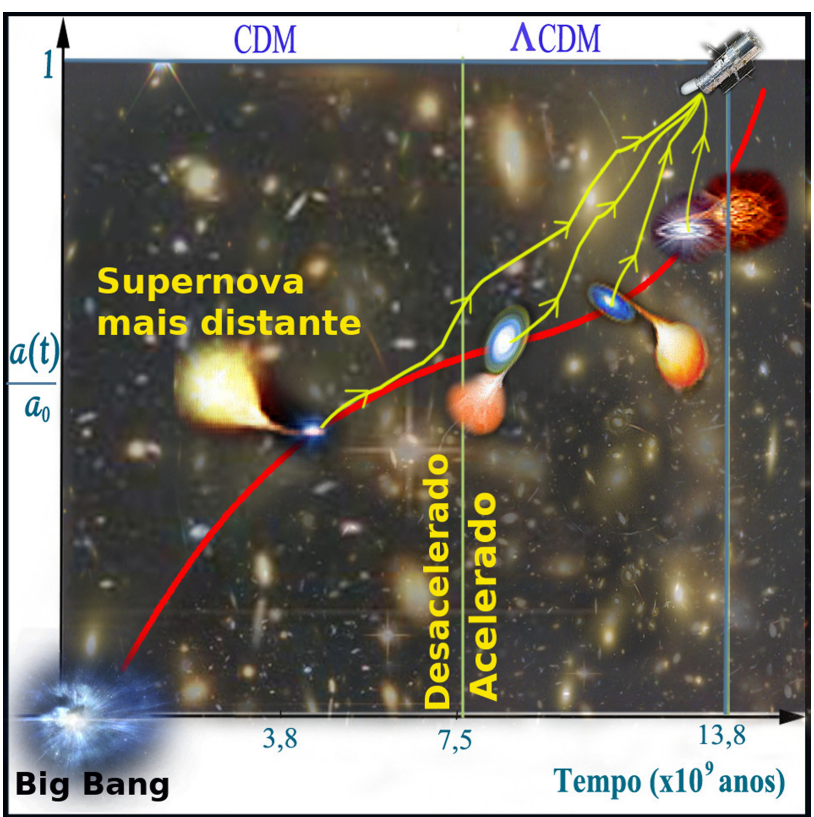

Figura 17: Supernovas em diferentes redshifts sofrendo o efeito de lente fraca ao longo do percurso. A primeira linha vertical separa a fase de expansão desacelerada (CDM) da fase acelerada $(\Lambda C D M)$. Já a segunda linha vertical indica o tempo presente. Note que a supernova mais distante foi imagiada quando o universo ainda estava na fase desacelerada.

técnica poderá gerar um conjunto de dados de SN Ia, proporcionando uma interessante sonda cosmológica no intervalo crítico de redshifts $1<z<2$.

\subsection{SNe Ia e Lentes Fortes}

Refsdal mostrou que a cosmografia com "time delay", envolve a diferença no tempo, $\Delta \tau$, de chegada da luz nas imagens múltiplas [42]. Combinando as medidas com um modelo para o potencial da lente, pode-se mostrar que o atraso depende de uma quantidade envolvendo as distâncias cosmológicas da fonte e da lente. Resulta que esta quantidade é inversamente proporcional a $H_{0} \mathrm{e}$ fracamente sensível aos outros parâmetros cosmológicos, de modo que $\Delta \tau \propto H_{0}^{-1}$. Portanto, medidas de time delay permitem inferir o valor da constante de Hubble. Alguns autores chamam esse método de TDSL (time delay strong lensing).

Estimativas de $H_{0}$ via TDSL são independente dos métodos locais, baseados na escada de distância cósmica (Cefeídas, Supernovas, etc.) ou em altos redshifts, via radiação cósmica de fundo, como as obtidas através dos satélites WMAP e Planck. Inicialmente, o TDSL fornecia valores de $H_{0}$ bem mais baixos do que os baseados nas Cefeídas. No entanto, o método foi empregado recentemente com relativo sucesso utilizando imagens múltiplas de quasares 79,80 . Fontes gravitacionalmente lenteadas com múltiplas imagens, provenientes de Supernovas (ou de quasares), podem fornecer uma valiosa contribuição para as medidas de $H_{0}$. 
Na Figura 18 mostramos a chamada Supernova de "Refsdal" e suas várias imagens. O redshift da SN é o mesmo da galáxia hospedeira $(z=1,49)$. Em destaque as quatro imagens (S1-S4) formando uma "Cruz de Einstein"[inserção (b)]. Na inserção (a) vemos a quinta imagem observada em 2015.

O método TDSL está sendo aplicado por pesquisadores envolvidos na colaboração COSMOGRAIL ${ }^{32}$ e HOLiCOW ${ }^{33}$ Neste ano, eles obtiveram um valor de $H_{0}$, relativamente alto e preciso, utilizando o TDSL com imagens de quasares. Um resultado que nos leva a discutir a tensão Supernova versus RCF, referente as medidas de $H_{0}$ e sua conexão com lentes gravitacionais.

\subsection{Tensão no Valor de $H_{0}$ e TDSL}

$\Lambda$ CDM plano é considerado o atual modelo cosmológico padrão. No entanto, desde que os resultados de diferentes experimentos cosmológicos cresceram em precisão, começaram a surgir alguns pontos de tensão. O mais notável e desafiador deles, é a discrepância entre as medições da constante de Hubble, a partir de análises da radiação cósmica de fundo (RCF) - pelas missões dos satélites WMAP e Planck - comparadas com as medidas locais, baseadas em Cefeídas e Supernovas, obtidas através do método padrão (diagrama de Hubble-Sandage).

Por um lado, as medidas de $H_{0}$ baseadas na RCF são oriundas de altos redshifts, atribuem um valor menor e são dependentes do modelo padrão, e do outro, os valores inferidos com base na escada de distância cósmica, embora dependentes da calibração, são locais, ou seja, obtidas em redshifts extremamente baixos. Neste caso,

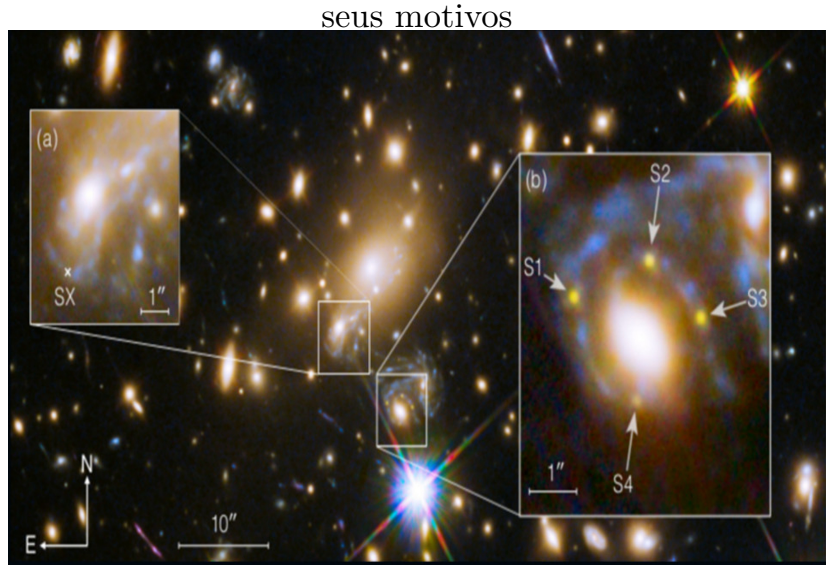

Figura 18: Descoberta da supernova "Refsdal" no aglomerado de galáxias, MACS J1149.6+2223. A galáxia hospedeira tem redshift $z=1,49$. Nos paineis vemos: (a) localização da quinta imagem detectada, observada pela primeira vez em dezembro de 2015, (b) primeiras quatro imagens, S1-S4, observadas quando a SN "Refsdal" foi detectada em 2014 [77. Disponível no artigo 78. (Crédito: NASA, ESA/Hubble).

\footnotetext{
${ }^{32}$ COSMOGRAIL $\equiv$ Cosmological Monitoring of Gravitational Lensing

${ }^{33} \mathrm{HOLiCOW} \equiv H_{0}$ Lensing in COSMOGRAIL's Wellspring
}

os valores de $H_{0}$ são maiores e independentes de modelo cosmológico.

A discrepância dos valores de $H_{0}$ obtida pelos dois métodos vem crescendo desde 2011. Paralelamente, a precisão nas medidas de $H_{0}$ por cada método também aumentou, tornando mais acentuada a divergência. Entre 2011 e 2014, as medidas locais chegaram a uma precisão melhor do que $3 \%$. Riess et al. 81 obtiveram $H_{0}=$ $73,8 \pm 2,4 \mathrm{~km} . \mathrm{s}^{-1} \cdot M p c^{-1}$ usando Cefeídas e Supernovas, enquanto Lima e Cunha [82] com a mesma precisão, obtiveram o valor $H_{0}=74,1 \pm 2,2 \mathrm{~km} . \mathrm{s}^{-1} \cdot M p c^{-1}$. Esse último resultado emergiu de uma análise combinando diferentes observações em redshifts intermediários $(\mathrm{z} \simeq$ 1); e tal como o Planck também depende do modelo $\Lambda \mathrm{CDM}$.

Na mesma época (2013), as colaborações do WMAP e Planck obtiveram, $H_{0}=70,0 \pm 2,2 \mathrm{~km} . \mathrm{s}^{-1} \cdot M p c^{-1} \mathrm{e}$ $H_{0}=67,4 \pm 1,4 \mathrm{~km} . \mathrm{s}^{-1} \cdot M p c^{-1}$, respectivamente, com incertezas de $\sim 2 \%$. Note que dentro das incertezas das medidas, os resultados da RCF são consistentes entre si, mas discrepantes com as medidas mais locais; sendo estatisticamente mais significante no caso do Planck. Nessa altura estava aceso o sinal vermelho entre astrônomos e cosmólogos. A discrepância em $H_{0}$ iria estacionar, aumentar ou diminuir?

Na Figura 19, mostramos a evolução das medidas de $H_{0}$ e o aumento contínuo da discrepância entre os 2 métodos. A discrepância aumentou entre 2015 e 2018, a medida que a precisão aumentava 84 86. Em 2019, utilizando dados de 70 Cefeídas imagiadas na grande

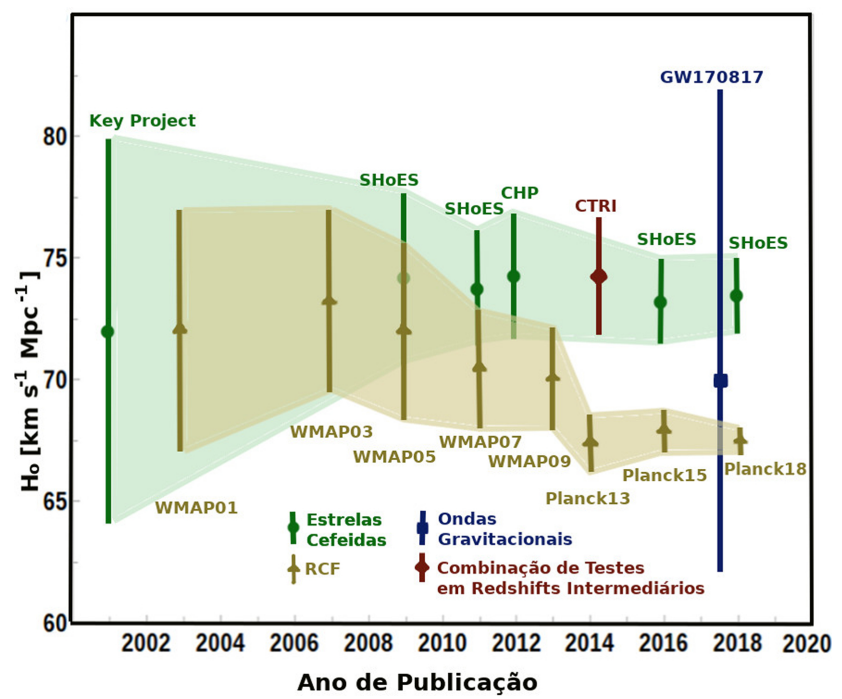

Figura 19: Evolução da Tensão em $H_{0}$. Pontos e região sombreada de verde foram obtidos com Cefeídas e Supernovas, enquanto os da região creme são determinados pelas medidas da RCF. O ponto em vermelho está relacionado a uma combinação de testes em redshifts intermediários (CTRI, Lima \& Cunha 2014 [82]). A barra azul é uma medida com ondas gravitacionais (adaptado de Freedman [83]). 
nuvem de Magalhães, a colaboração SH0ES 34 obteve $H_{0}=74,03 \pm 1,42 \mathrm{~km} . s^{-1} \cdot M p c^{-1}$; valor a ser comparado com $H_{0}=67,4 \pm 0,5 \mathrm{~km} . s^{-1} \cdot M p c^{-1}$, publicado pelo Planck (2018). Tais resultados implicam que a diferença entre os valores de $H_{0}$ medido localmente e pelo Planck, $\Delta H_{0}=6,6 \pm 1,5 \mathrm{~km} . \mathrm{s}^{-1} \cdot M p c^{-1}$, bem além de uma discrepância meramente aleatória $(4.4 \sigma)$ [87]. A discrepância parece consolidada, mas sua explicação continua desconhecida.

Qual será o valor correto de $H_{0}$, o de altos ou de baixos redshifts? Mais importante no presente contexto: Qual o papel das lentes gravitacionais nessa crise?

Cosmografia de "time delay" (TDSL) usa atrasos de tempo de lente gravitacional e pode medir a constante de Hubble. Como observado no final da seção anterior, a idéia vem sendo implementada no âmbito do projeto H0LiCOW 88 em colaboração com o COSMOGRAIL [89]. Como resultado, supondo um modelo $\Lambda \mathrm{CDM}$ plano, obtiveram para o QSO $1206+4332$ com duas imagens, o seguinte valor: $H_{0}=72,5_{-2,3}^{+2,1} \mathrm{~km} . s^{-1} . M p c^{-1}$ com precisão de $3 \%$. Uma medida independente da escada de distância cósmica e de outros testes cosmológicos, mas dependente do modelo $\Lambda$ CDM plano [90].

Não sabemos se o resultado acima resolve a tensão sobre o valor correto de $H_{0}$. Talvez um solução definita venha com o telescópio LSST ${ }^{35}$ que está sendo construído no Chile. Dotado de um espelho de 8,4 metros e projetado para entrar em operação em 2022, o LSST será capaz de mapear todo o céu visível. Com o LSST, espera-se obter milhares de transientes com lentes fortes (supernovas e quasares) que deverão turbinar e aumentar a precisão da cosmografia do atraso de tempo.

\section{Lentes e Ondas Gravitacionais: O Futuro?}

Recentemente, as colaborações LIGO ${ }^{36}$ e VIRGO ${ }^{37}$ detectaram ondas gravitacionais (OGs) emitidas durante a fusão de pares de buracos negros e choque entre estrelas de nêutrons 91 93. Esta bem sucedida previsão da relatividade geral, pressiona por esforços observacionais envolvendo a próxima geração de detectores de OGs no solo e no espaço, planejados para "ouvir" bandas de frequência emitidas por uma grande variedade de fontes. Numa astronomia multimensageira, é natural que a interface unindo lentes e OGs seja também investigada.

$\mathrm{Na}$ relatividade geral, grávitons são partículas sem massa e, tal como os fótons, seguem geodésicas nulas. Isto significa que o campo gravitacional deverá também agir como um meio refrator sobre as OGs (ver Apêndice A). Como acontece com feixes de luz, discutidos na

\footnotetext{
${ }^{34}$ SHOES $\equiv$ Supernovae, $H_{0}$, for the Equation of State of Dark Energy

${ }^{35} \mathrm{LSST} \equiv$ Large Synoptic Survey Telescope.

${ }^{36}$ LIGO $\equiv$ Laser Interferometer Gravitational-Wave Observatory

${ }^{37}$ VIRGO $\equiv$ Interferômetro Europeu de Ondas Gravitacionais
}

aproximação da ótica geométrice 38 os feixes de grávitons serão também defletidos.

No entanto, a escala macroscópica característica das lentes gravitacionais é o raio gravitacional $\left(L=R_{g}=\right.$ $\left.2 G M / c^{2}\right)$. Isto significa que o limite da ótica geométrica gravitacional $\left(\lambda_{g}<<R_{g}\right)$, pode não ser satisfeito em muitas situações. Por exemplo, no caso do LIGO $\left(10 \mathrm{~km}<\lambda_{g}<10^{4} \mathrm{~km}\right)$, a ótica geométrica será apropriada quando a lente for uma galáxia, mas não um objeto ultracompacto, pois neste caso $\lambda_{g} \gtrsim R_{g}$. Portanto, efeitos ondulatórios como a difração, em geral, não poderão ser ignorados, tornando a fenomenologia do lenteamento das OGs bem mais rica e complexa.

Ondas gravitacionais são perturbações no espaço-tempo, potencialmente, afetando o campo da lente e suas imagens. No ótico, a perturbação da onda pode afetar as "cintilações"alterando o "time-delay" entre a fonte e as imagens. Para o quasar duplo $0957+561$ (A,B), o atraso medido é de 415 dias. Isto significa que possíveis contribuições de ondas gravitacionais podem estar incluídas no processo e precisam ser subtraídas.

Por outro lado, se imagens gravitacionais múltiplas forem identificadas, outras possibilidades serão abertas. Por exemplo, parâmetros cosmológicos medidos via OGs com o efeito de "sirenes cósmicas" 94, 95, fornecerão uma estratégia alternativa de solução para a presente tensão sobre a constante de Hubble. O caso mais óbvio podemos denominar GTDSL (Gravitational Time Delay Strong Lensing), em perfeita analogia com o TDSL.

Similarmente, sistemas exoplanetários de período ultracurtos são também fontes interessantes de ondas gravitacionais para a próxima geração de detectores, pois estão dentro da curva de sensibilidade do LISA 39 e outros instrumentos planejados. Reciprocamente, as ondas gravitacionais emitidas podem fornecer uma janela complementar para identificar planetas extrasolares via microlentes gravitacionais, um novo procedimento que pode ser ainda mais eficiente do que os métodos disponíveis (como o tempo de trânsito) baseados em instrumentos e técnicas ópticas 96, 97.

\section{Comentários Finais \& Perspectivas}

Um século depois da legendária observação do eclipse solar total, confirmando o resultado da deflexão relativística prevista por Einstein, podemos afirmar que as lentes gravitacionais constituem uma área de pesquisa consolidada da Astronomia.

$\mathrm{Na}$ verdade, no final dos anos 80, a lista de descobertas científicas em lentes gravitacionais ainda era modesta, embora os progressos realizados até meados da idade moderna já fossem animadores. Em outras palavras, apesar dos inúmeros obstáculos, valia a pena investigar as aplicações de lentes gravitacionais; uma área oferecendo novas técnicas na abordagem de problemas fundamentais [4]. De fato, além de proporcionar vários "efeitos

\footnotetext{
38 Ver rodapé 9.

${ }^{39}$ LISA $\equiv$ Laser Interferometer Space Antenna
} 


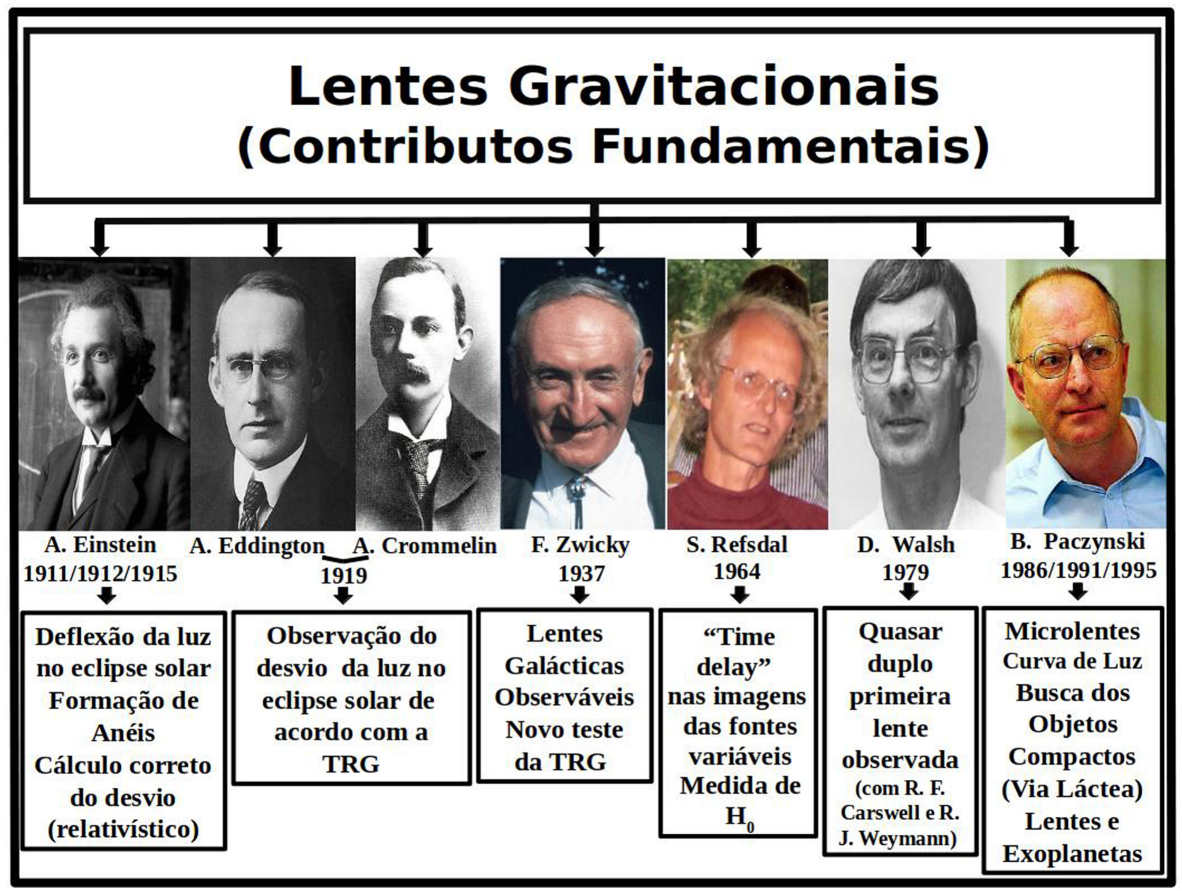

Figura 20: Fundadores da área de lentes gravitacionais (Idade Antiga e Moderna). O período entre 1938-1962, foi a idade média das lentes gravitacionais. Seu renascimento ocorreu nos anos 60-70 com os trabalhos teóricos de Refsdal e a descoberta do quasar duplo 0957 + 561 A \& B (infelizmente, os autores não encontraram fotos de Carswell e Weymann). Tais resultados foram consolidados pelas diferentes aplicações da curva de luz de Paczinski, na sua mais criativa década.

especiais" como os anéis e a cruz de Einstein, a magnificação variável da curva de luz de estrelas fontes e a formação de arcos gigantes em aglomerados, revelou-se também uma disciplina com ramificações em várias direções; desde a busca dos objetos escuros até as mais desafiadoras questões cosmológicas.

$\mathrm{Na}$ Figura 20 mostramos os pioneiros da teoria de lentes gravitacionais das eras antiga e moderna (cf. Figuras 1 e 8. Passadas as fases mais heróicas, digamos, até meados dos anos 80, estamos agora vivenciando a era da precisão observacional. Entramos numa fase de projetos e colaborações de grande envergadura, tanto em recursos humanos e financeiros quanto em sofisticação tecnológica. Isto significa que os objetivos científicos são bem definidos, mas a coleta e o tratamento de dados revela-se uma tarefa colossal, tornando todo e qualquer avanço um trabalho de caráter bem mais coletivo. Por isso evitamos uma cronologia pontuando nomes na fase contemporânea.

Naturalmente, a nova realidade não exclui o exercício individual da criatividade, nem tão pouco a possibilidade de uma quebra de paradigma na disciplina. Nesse sentido, uma atenção especial deverá ser dedicada as novidades oriundas dos campos emergentes envolvendo lentes, desde a busca por exoplanetas até as ondas gravitacionais.

Descobrir novos planetas é um dos grandes objetivos da astronomia moderna com consequências para o estudo da origem e preservação da vida em diferentes ambientes do Universo. A busca por planetas extrasolares na chamada zona habitáve ${ }^{40}$ tem sido reforçada por satélites exclusivamente dedicados a tarefa. Nessa conexão, microlentes hoje é parte do conjunto de técnicas utilizadas nas descobertas, tais como: imagiamento direto, tempo de trânsito e as medidas de velocidade radial. Mais importante ainda, microlentes explora regimes de massas e tamanhos de órbitas diferentes das outras técnicas (seção 4.1).

Sem dúvida, podemos afirmar que a área de lentes gravitacionais tem tido um sucesso espetacular no estudo de fenômenos astronômicos; varrendo desde objetos individuais, como planetas, estrelas, galáxias e aglomerados, até a distribuição de massa em várias escalas; incluindo ainda medidas de vários parâmetros cosmológicos e uma possível solução da tensão envolvendo as medidas da constante de Hubble em altos e baixos redshits.

Todas essas realizações garantem expectativas extremamente positivas para os próximos vinte anos. Nesse contexto, não será surpreendente se a detecção futura do lenteamento das ondas gravitacionais abrir uma nova janela de oportunidades para nossa compreensão do Cosmos.

\section{Agradecimentos}

JASL agradece ao CNPq, CAPES (PROCAD 2013) e FAPESP (Projeto LLAMA).

\footnotetext{
${ }^{40} \mathrm{Um}$ planeta está na zona habitável quando sua distância até a estrela hospedeira é compatível com a existência de água líquida.
} 


\section{Material suplementar}

O seguinte material suplementar está disponível online: Apêndice A: Campo Gravitacional como um Meio Refrator.

Apêndice B: Magnificação e Variabilidade.

\section{Referências}

[1] A.S. Eddington, The Observatory 42, 119 (1919).

[2] A. Pais, Sútil é o Senhor, Vida e Pensamento de Albert Einstein (Gradiva, Lisboa, 1982), p. 372.

[3] L.C.B. Crispino e D.J. Kennefick, Nature Physics 15, 416 (2019).

[4] E.L. Turner, Scientific American 259, 26 (1988).

[5] P. Schneider, J. Ehlers e E.E. Falco, Gravitational Lenses: Strong, Weak and Micro (Springer, Berlim, 1999), v. 33, $2^{\mathrm{a}}$ ed.

[6] S. Mollerach e E. Roulet, Gravitational Lensing and Microlensing (World Scientific, Singapore, 2002).

[7] A.B. Congdon e C.R. Keeton, Principles of Gravitational Lensing (Springer, New York, 2018).

[8] R. Narayan e M. Bartelmann, arXiv:astro-ph/9606001 (1996).

[9] P. Schneider, C. Kochanek e J. Wambsganss, Gravitational Lensing: Strong, Weak and Micro (Springer, Berlim, 2006).

[10] M. Bartelmann, Class. and Quant. Grav. 27, 23 (2010).

[11] Y. Tsapras, Geosciences 8, 365 (2018).

[12] H.M. Nussenzveig, in: Progress in Optics (Elsevier, New York, 2007), v. 50, p. 185.

[13] R.A. Martins e C.C. Silva, Revista Brasileira de Ensino de Física 37, 4202 (2015).

[14] I. Newton, Optikcs or A Treatise of the Reflections, Refractions, Inflections or Colors of Light (William Innys, London, 1704).

[15] J. Michel, Philo. Trans. R. Soc. Lon. 74, 35 (1784).

[16] P.S. Laplace, Exposition du Systéme du Monde (De I'Imprimerie du Circle Social, Paris, 1796), v. 2, p. 305.

[17] J.G. von Soldner, Berliner Astronomisches Jahrbuch, 161 (1801).

[18] L.D. Landau e E.M. Lifshitz, The Classical Theory of Fields (Pergamon Press, New York, 1975), p. 301.

[19] C.M. Will, Amer. J. Phys. 56, 413 (1988).

[20] A. Einstein, in: Textos Fundamentais da Física Moderna (Fundação Calouste Gulbenkian, Lisboa, 1971), p. 47.

[21] H. Poincaré, C. R. Acad. Sci. Paris 14, 1504 (1905).

[22] A. Einstein, Jahrb. Radioakt. ElektroniK 4, 411 (1907).

[23] A. Einstein, O Princípio da Relatividade (Fundação Calouste Gulbenkian, Lisboa, 1971), v. 1, p. 99.

[24] F.W. Dyson, A.S. Eddington e C.R. Davidson, Mem. Roy. Astron. Soc. 62, 291 (1920).

[25] D.E. Lebach, B.E. Corey, I.I. Shapiro, M.I. Ratner, J.C. Webber, A.E. Rogers, J.L. Davis e T.A. Herring, Phys. Rev. D 75, 1439 (1995).

[26] O. Chwolson, Astr. Nachrichten 221, 329 (1924).

[27] A. Einstein, Science 84, 506 (1936).

[28] J. Renn, T. Sauer e J. Stachel, Science 275, 184 (1997).

[29] F. Zwicky, Phys. Rev. 51, 290 (1937).

[30] F. Zwicky, Phys. Rev. 51, 679 (1937).

[31] J.A.S. Lima e J.S. Alcaniz, Astron. Astrophys. 357, 393 (2000).
[32] R.C. Santos, Efeitos das inomogeneidades da matéria em Cosmologias Aceleradas. Tese de Doutorado, Universidade Federal do Rio Grande do Norte, Natal (2007).

[33] R.C. Santos e J.A.S. Lima, Phys. Rev. D 77, 083505 (2008).

[34] J.A.S. Lima e R.C. Santos, Revista Brasileira de Ensino de Física 40, e1313 (2018).

[35] H. Ebiling, M. Stockmann, J. Richard, J. Zabl, G. Brammer, S. Toft e A. Man, Astrophys. J. 852, L7 (2018).

[36] W.L. Burke, Astrophys. J. Lett. 244, 1 (1981).

[37] R.H. McKenzie, JMP 26, 1592 (1985).

[38] R. Lynds e V. Petrosian, BAAS 18, 1014 (1986).

[39] J.A. Tyson, F. Valdes e R.A. Wenk, Astrophys. J. 349, L1 (1990).

[40] M. Schmidt, Nature 197, 1040 (1963).

[41] S. Refsdal, MNRAS 128, 295 (1964).

[42] S. Refsdal, MNRAS 128, 307 (1964).

[43] D. Walsh, R.F. Carswell e R.J. Weymann, Nature 279, 381 (1979).

[44] J. Hewitt, Nature 333, 537 (1988).

[45] P. Young, J.E. Gunn, J.B. Oke, J.A. Westphal e J. Kristian, Astrophys. J. 241, 507 (1980).

[46] K.C. Freeman, Astrophys. J. 161, 802 (1970).

[47] V.C. Rubin e W.K. Ford Jr., Astrophys. J. 159, 379 (1970).

[48] V.C. Rubin, Science 220, 1339 (1983).

[49] V.C. Rubin, Scientific American 248, 88 (1983).

[50] J.P. Ostriker e P.J.E. Peebles, Astrophys. J. 186, 467 (1973).

[51] P.J.E. Peebles, Principles of Physical Cosmology (Princeton University Press, New Jersey, 1993), p. 47.

[52] B.K. Stoychev, arXiv:1905.00432 (2019).

[53] B. Paczynski, Astrophys. J. 301, 503 (1986).

[54] B. Paczynski, Astrophys. J. 304, 1 (1986).

[55] J.P. Beaulieu, D.P. Bennett, P. Fouqué, A. Williams, M. Dominik, U.G. Jorgensen, D. Kubas, A. Cassan, C. Coutures, J. Greenhill, et al., Nature 439, 437 (2006).

[56] K.C. Sahu, in: Proceedings of the STScI Symposium on "Dark Universe: Matter, Energy, and Gravity (Cambridge University Press, Cambridge, 2003), p. 14.

[57] B. Paczynski, Ann. Rev. Astron. Astrophys. 34, 419 (1996).

[58] S. Mao, B. Paczynski e M. Jaroszynski, Astrophys. J.374, L37 (1991).

[59] I.A. Bond, A. Udalski, N.J. Rattenbury, B. Paczyński, I. Soszyński, L. Wyrzykowski, M.K. Szymański, M. Kubiak e O. Szewczyk, Astrophys. J. 606, L155 (2004).

[60] E.S. Cypriano, L. Sodré Jr., J.P. Kneib e L.E. Campusano, Astrophys. J. 613, 95 (2004).

[61] M. Meneghetti, M. Bartelmann, H. Dahle e M. Limousin, Space Sci. Rev. 177, 31 (2013).

[62] S. Dodelson, Gravitational Lensing (Cambridge University Press, Cambridge, 2017).

[63] F. Zwicky, Helvetica Phys. Acta 6, 110 (1933).

[64] D. Clowe, M. Bradač, A.H. Gonzalez, M. Markevitch, S.W. Randall, C. Jones e D. Zaritsky, Astrophys. J. Lett., 648, L109 (2006).

[65] R. Monteiro-Oliveira, E.S. Cypriano, A.Z. Vitorelli, A.L.B. Ribeiro, L. Sodré Jr, R. Dupke e C.M. Oliveira, MNRAS 481, 1097 (2018). 
[66] M. Markevitch, A.H. Gonzalez, D. Clowe, A. Vikhlinin, L. David, W. Forman, C. Jones, S. Murray e W. Tucker, in: Multiwavelength Cosmology editado por M. Plionis (Academic Publishers, Kolkata, 2004).

[67] M. Milgrom, Astrophys. J. 270, 365 (1983).

[68] A. Aguirre, J. Schaye e E. Quataert, Astrophys. J. 561, 550 (2001).

[69] A.G. Riess, A.V. Filippenko, P. Challis, A. Clocchiatti, A. Diercks, P.M. Garnavich, R.L. Gilliland, C.J. Hogan, S. Jha e R.P. Kirshner, Astron. J. 116 1009, (1998).

[70] S. Perlmutter, G. Aldering, G. Goldhaber, R.A. Knop, P. Nugent, P.G. Castro, S. Deustua, S. Fabbro, A. Goobar, D.E. Groom et al., Astrophys. J. 517, 565 (1999).

[71] A. Albrecht, G. Bernstein, R. Cahn, W.L. Freedman, J.Hewitt, W.Hu, J. Huth, M. Kamionkowski, E.W. Kolb, L. Knox et al., arXiv:astro-ph/0609591 (2006).

[72] Y. Wang, JCAP 2005, 005 (2005).

[73] R. Mandelbaum, ARAA 56, 393 (2017).

[74] M. Goliath e E. Mörtsell, Phys. Lett. B 486, 249 (2000).

[75] J. Jönsson, T. Kronborg, E. Mörtsell e J. Sollerman, Astron. \& Astrophys. 487, 467 (2008).

[76] M. Smith, D.J. Bacon, R.C. Nichol, H. Campbell, C. Clarkson, R. Maartens, C.B. D'Andrea, B.A. Bassett, D. Cinabro, D.A. Finley et al., Astrophys. J. 780, 24 (2014).

[77] P.I. Kelly, S.A. Rodney, T. Treu, R.J. Foley, G. Brammer, K.B. Schmidt, A. Zitrin, A. Sonnenfeld, L.G. Strolger, O. Graur et al., Science 347, 1123 (2015).

[78] T. Treu e R.S. Ellis, Contemp. Phys. 56, 17 (2015).

[79] T. Treu, G. Brammer, J.M. Diego, C. Grillo, P.L. Kelly, M. Oguri, S.A. Rodney, P. Rosati, K. Sharon, A. Zitrin et al., Astrophys. J. 817, 60 (2016).

[80] C. Grillo, P. Rosati, S.H. Suyu, I. Balestra, G.B. Caminha, A. Halkola, P.L. Kelly, M. Lombardi, A. Mercurio, A. Rodney et al., The Astrophysical Journal 860, 94 (2018).

[81] A.G. Riess, L. Macri, S. Casertano, H. Lampeitl, H.C. Ferguson, A.V. Filippenko, S.W. Jha, W. Li e R. Chornock, Astrophys. J 732, 129 (2011).

[82] J.A.S Lima e J.V. Cunha, Astrophys. J. 781, 2 (2014).

[83] W.L. Freedman, Nat. Astron. 1, 0121 (2017).

[84] Planck Collaboration, Astron. \& Astrophys. 594, A13 (2016).

[85] A.G. Riess, L.M. Macri, S.L. Hoffmann, D. Scolnic, S. Casertano, A.V. Filippenko, B.E. Tucker, M.J. Reid, D.O. Jones, J.M. Silverman et al., Astrophys. J. 826, 56 (2016).

[86] Planck Collaboration, arXiv:1807.06209 (2018).

[87] A.G. Riess, L.M. Macri, S.L. Hoffmann, D. Scolnic, S. Casertano, A.V. Filippenko, B.E. Tucker, M.J. Reid, D.O. Jones, J.M. Silverman et al., Astrophys. J. 876, 85 (2019).

[88] S.H. Suyu, V. Bonvin, F. Courbin, C.D. Fassnacht, C.E. Rusu, D. Sluse, T. Treu, K.C. Wong, M.W. Auger, X. Ding et al., MNRAS 468, 2590 (2017).

[89] M. Tewes, F. Courbin e G. Meylan, Astron. Astrophys 609, A71 (2018).

[90] S. Birrer, T. Treu, C.E. Rusu, V. Bonvin, C.D. Fassnacht, J.H.H. Chan, A. Agnello, A.J. Shajib, G.C.F Chen, M. Auger et al., MNRAS 484, 4726 (2019).

[91] B.P. Abbott, R. Abbott, T.D. Abbott, S. Abraham, F. Acernese, K. Ackley, C. Adams, R.X. Adhikari, V.B.
Adya, C. Affeldt et al., Phys. Rev. Lett. 116, 061102 (2016).

[92] B.P. Abbott, R. Abbott, T.D. Abbott, S. Abraham, F. Acernese, K. Ackley, C. Adams, R.X. Adhikari, V.B. Adya, C. Affeldt, et al., Phys. Rev. Lett. 116, 241103 (2016).

[93] B.P. Abbott, R. Abbott, T.D. Abbott, F. Acernese, K. Ackley, C. Adams, T. Adams, P. Addesso, R.X. Adhikari, V.B. Adya et al., Phys. Rev. Lett. 118, 221101 (2017).

[94] K. Liao, F. Xi-Long, X. Ding, M. Biesiada e Z. ZongHong, Nature Communications 8, 1148 (2017).

[95] S.M. Feeney, H.V. Peiris, A.R. Williamson, S.M. Nissanke, D.J. Mortlock, J. Alsing e D. Scolnic, Phys. Rev. Let. 122, 061105 (2019).

[96] J.V. Cunha, F.E. Silva e J.A.S. Lima, MNRAS 480, L28 (2018).

[97] N. Tamanini e C. Danielski, The gravitational wave detection of exoplanets orbiting white dwarf binaries using LISA, disponível em https://www.nature.com/articles/s41550-019-0807-y. 